\title{
REVIEW ARTICLE OPEN The molecular biology of pancreatic adenocarcinoma: translational challenges and clinical perspectives
}

\author{
Shun Wang ${ }^{1}$, Yan Zheng ${ }^{1}$, Feng Yang ${ }^{2}$, Le Zhu ${ }^{1}$, Xiao-Qiang Zhu ${ }^{3}$, Zhe-Fang Wang ${ }^{4}$, Xiao-Lin Wu ${ }^{4}$, Cheng-Hui Zhou ${ }^{4}$, Jia-Yan Yan ${ }^{4,5}$,
} Bei-Yuan Hu${ }^{1}$, Bo Kong ${ }^{6}$, De-Liang Fu' ${ }^{2}$, Christiane Bruns ${ }^{4}$, Yue Zhao ${ }^{4}$, Lun-Xiu Qin ${ }^{1}$ and Qiong-Zhu Dong (iD ${ }^{1,7}$

Pancreatic cancer is an increasingly common cause of cancer mortality with a tight correspondence between disease mortality and incidence. Furthermore, it is usually diagnosed at an advanced stage with a very dismal prognosis. Due to the high heterogeneity, metabolic reprogramming, and dense stromal environment associated with pancreatic cancer, patients benefit little from current conventional therapy. Recent insight into the biology and genetics of pancreatic cancer has supported its molecular classification, thus expanding clinical therapeutic options. In this review, we summarize how the biological features of pancreatic cancer and its metabolic reprogramming as well as the tumor microenvironment regulate its development and progression. We further discuss potential biomarkers for pancreatic cancer diagnosis, prediction, and surveillance based on novel liquid biopsies. We also outline recent advances in defining pancreatic cancer subtypes and subtype-specific therapeutic responses and current preclinical therapeutic models. Finally, we discuss prospects and challenges in the clinical development of pancreatic cancer therapeutics.

Signal Transduction and Targeted Therapy (2021)6:249

; https://doi.org/10.1038/s41392-021-00659-4

\section{INTRODUCTION}

Pancreatic cancer, with one of the highest mortality rates of all malignancies, is the seventh leading cause of cancer-related death worldwide. ${ }^{1}$ With almost as many deaths $(n=466,000)$ as new cases ( $n=496,000)$ according to GLOBOCAN 2020 estimates, ${ }^{1}$ pancreatic cancer has become the third leading cause of cancerrelated death in the United States. ${ }^{2}$ Despite improvements in surgical techniques and therapy regimens, the 5-year survival rate for pancreatic cancer is still the lowest $(9 \%)$ of any cancer type. ${ }^{3,4}$

Approximately $90 \%$ of pancreatic cancers are characterized as pancreatic ductal adenocarcinoma (PDAC). Risk factors associated with pancreatic cancer include familial risk due to susceptibility gene mutations, chronic pancreatitis, pancreatic cysts, and diabetes mellitus., ${ }^{5,6}$ Other risk factors include smoking, alcohol abuse, obesity or metabolic syndrome, aging, and occupational exposure. ${ }^{4,7}$ Approximately $80 \%$ of pancreatic cancer patients present with advanced disease or distant metastases and have no effective treatment options. ${ }^{8}$ Even early-stage patients eligible for resection have a 5 -year survival rate of $<31 \% .^{9}$ Advances in nextgeneration genome sequencing (NGS) have brought new excitement to the field by supporting the identification of molecular alterations that govern pancreatic cancer progression. Sequencing data have revealed that pancreatic cancer comprises highly heterogeneous tumors that develop resistance to traditional chemotherapy and radiation therapy.

Gemcitabine has been a reference first-line therapeutic drug for pancreatic cancer patients since $1997 .{ }^{10}$ However, traditional chemotherapy and radiation therapy have not shown improved 5year survival rates. ${ }^{11,12}$ Modified FOLFIRINOX (mFOLFIRINOX) (i.e., 5-fluorouracil (5-FU), leucovorin, oxaliplatin, and irinotecan) and nab-paclitaxel plus gemcitabine are generally considered as the best adjuvant chemotherapy regimens, although they have shown only modest survival benefits with considerable toxicity. ${ }^{13}$ Furthermore, multiple novel-targeted therapies (i.e., cetuximab, bevacizumab, axitinib, and aflibercept) have failed to effectively improve overall survival (OS), while erlotinib combined with gemcitabine showed a marginal clinical benefit. ${ }^{14,15}$ Therefore, new therapies, e.g., innovative immunotherapies and combination therapies with increased antitumor potency, are urgently needed.

In this review, we first provide a general discussion of pancreatic cancer. This is followed by a review of novel biomarkers for their diagnosis, treatment monitoring, and prognosis based on liquid biopsy. We conclude with a discussion of the clinical perspectives of current advances. We anticipate that novel therapeutic methods and strategies based on subtype-specific therapy and preclinical therapeutic models might have a great impact on pancreatic cancer treatment and management, thereby improving patient outcomes.

\section{CHARACTERISTICS OF PANCREATIC CANCER}

Genetics and epigenetics of pancreatic cancer

Via the use of NGS and computational biology, we have gained a deeper understanding of the genetic alterations underlying the

\footnotetext{
${ }^{1}$ Department of General Surgery, Huashan Hospital, Cancer Metastasis Institute, Fudan University, Shanghai, China; ${ }^{2}$ Department of Pancreatic Surgery, Pancreatic Disease Institute, Huashan Hospital, Fudan University, Shanghai, China; ${ }^{3}$ School of Biomedical Sciences, Li Ka Shing Faculty of Medicine, The University of Hong Kong, Hong Kong, China; ${ }^{4}$ General, Visceral and Cancer Surgery, University Hospital of Cologne, Cologne, Germany; ${ }^{5}$ Department of Biliary-Pancreatic Surgery, Renji Hospital, School of Medicine, Shanghai Jiao Tong University, Shanghai, China; ${ }^{6}$ Department of Surgery, Klinikum rechts der Isar, School of Medicine, Technical University of Munich (TUM), Munich, Germany and ${ }^{7}$ Key laboratory of whole-period monitoring and precise intervention of digestive cancer, Shanghai Municipal Health Commission (SMHC), Shanghai, China

Correspondence: Yue Zhao (yue.zhao@uk-koeln.de) or Lun-Xiu Qin (qinlx@fudan.edu.cn) or Qiong-Zhu Dong (qzhdong@fudan.edu.cn)

These authors contributed equally: Shun Wang, Yan Zheng
}

Received: 26 February 2021 Revised: 27 April 2021 Accepted: 26 May 2021

Published online: 05 July 2021 


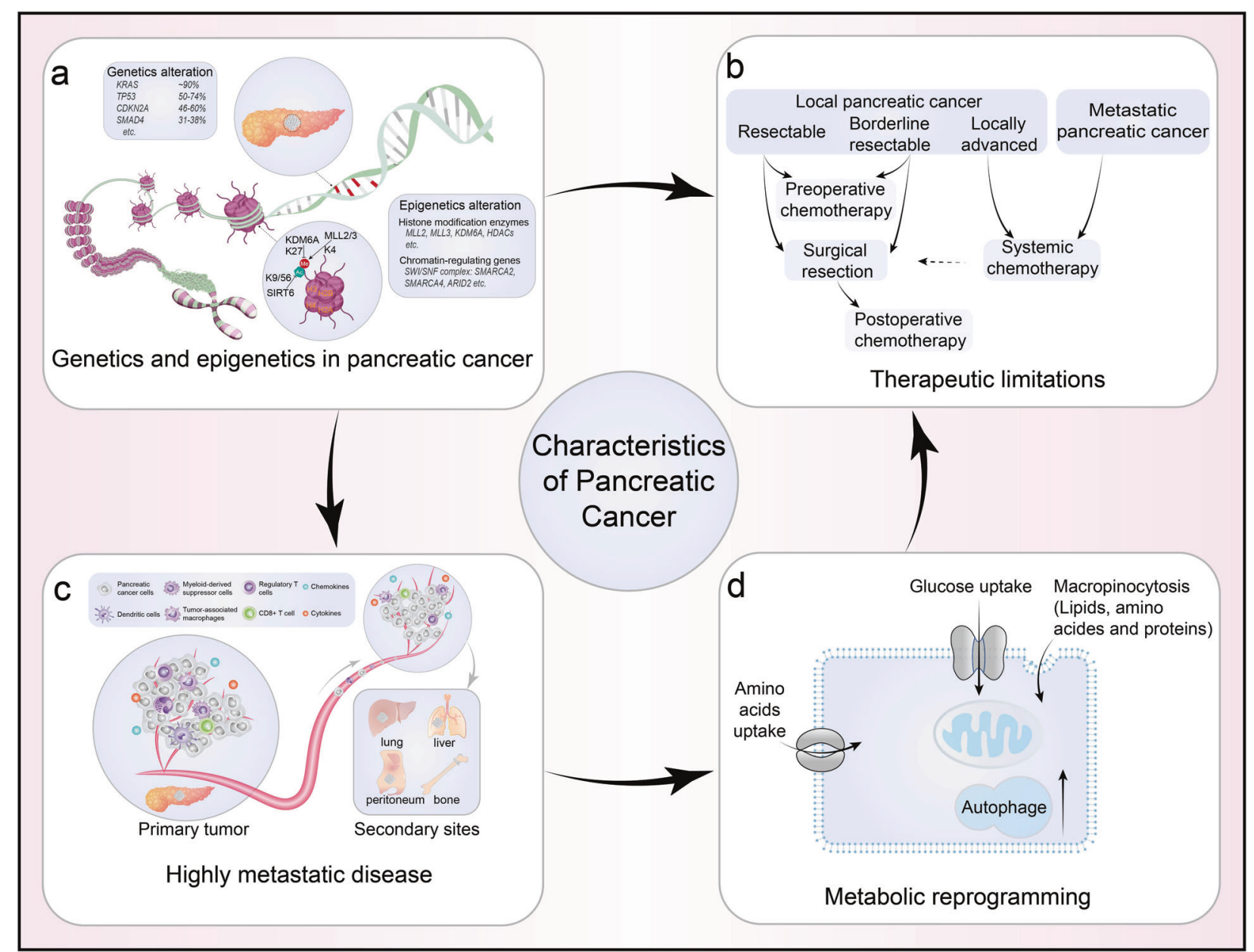

Fig. 1 The characteristics of pancreatic adenocarcinoma. Pancreatic cancer is a common cause of cancer mortality characterized by high heterogeneity, a dense stromal tumor microenvironment (TME), highly metastatic propensity, metabolic reprogramming, and limited benefits from current conventional therapies. a Genetic and epigenetic changes in pancreatic cancer. KRAS ( 90\%), TP53 (50-74\%), CDKN2A (46-60\%), and SMAD4 (31-38\%) are the most frequently mutated genes known to modulate the initiation and progression of pancreatic cancer. Epigenetic regulatory genes, including MLL2/3, KDM6A, and multiple HDACs encoding genes, regulate histone modification. SMARCA2/4 and ARID2 modulate chromatin remodeling. b Therapeutic limitations in pancreatic cancer. Surgical resection is the only potentially curative choice for pancreatic cancer patients. Adjuvant chemotherapy can only partially improve the overall survival of pancreatic cancer patients c Pancreatic cancer is an extremely aggressive tumor with high metastatic propensity. The immunosuppressive TME plays an important role in modulating the metastasis of pancreatic cancer cells to the liver, lungs, peritoneum, and bone. $\mathbf{d}$ Metabolic reprogramming of pancreatic cancer. Pancreatic cancer cells can survive and proliferate in stressful microenvironments by reprogramming energy metabolism to increase glucose and glutamine uptake, macropinocytosis, and autophagy

genesis and progression of pancreatic cancer, including gene expression changes, copy number aberrations, chromosomal rearrangements, and epigenetic alterations (Fig. 1a). Pancreatic cancer typically starts as a precancerous lesion, i.e., a pancreatic intraepithelial neoplasia (PanIN), which accumulates gene mutations over time, ultimately progressing to a more dysplastic state. $^{16-18}$ Approximately $90 \%$ of pancreatic cancers of all grades have activating oncogenic Kirsten rat sarcoma viral oncogene homolog (KRAS) mutations. Among the oncogenic KRAS mutations associated with pancreatic cancer in humans, the GAT (aspartic acid; G12D), GTT (valine; G12V), and TGT (cysteine; G12C) mutations are the most common, while the CGT (arginine (Arg); G12R) and GCT (alanine; G12A) mutations as well as other point mutations at codons $11,13,61$, or 146 appear to be less common. ${ }^{17,18}$ As the most common activating mutations in pancreatic cancer, KRAS mutations (which have also been investigated in depth in various other cancers, including metastatic colorectal cancer and non-small cell lung cancer) impair the intrinsic GTPase activity of KRAS and prevent GTPase-activating proteins (GAPs) from converting the active GTP-bound form to the
GDP-bound inactive form. Clinical studies have also shown that KRAS mutations could be considered as a marker for the poor prognosis of pancreatic cancer. ${ }^{17}$ However, it has been reported that various KRAS mutations affect diverse signaling pathways, leading to distinct functional consequences. Pancreatic cancer patients with KRAS at codon 61 mutations exhibit lower extracellular signal-regulated kinase (ERK) activation compared with patients with other KRAS alleles, and the former harbor significantly better prognosis. ${ }^{19-21}$ It is evident that KRAS mutation seems to be necessary but not sufficient for pancreatic cancer development. Other genes, including tumor protein p53 (TP53), cyclin-dependent kinase inhibitor $2 \mathrm{~A}(C D K N 2 A)$, and SMAD family member 4 (SMAD4), are also frequently involved in pancreatic cancer tumorigenesis and metastasis. ${ }^{22}$ Approximately $50-74 \%$ of pancreatic cancers have inactivating mutations in the tumor suppressor gene TP53. ${ }^{16,23}$ TP53 inactivation impairs DNA damage recognition and blocks cell cycle arrest, allowing cells to bypass cell cycle checkpoints and evade apoptotic signals. ${ }^{16,23}$ Mutations in CDKN2A, which can lead to loss of regulation of the cyclindependent kinase (CDK) 4 and CDK6 cell cycle checkpoints and, 
thus, dysregulation of the cell cycle and subsequent carcinogenesis, are detected in $\sim 46-60 \%$ of pancreatic cancers. ${ }^{16,23,24}$ In addition, $\sim 31-38 \%$ of pancreatic cancers have mutations in SMAD4, which can occur in the late stages of pancreatic carcinogenesis. ${ }^{23,25}$ In pancreatic cancer, the frequent loss of $S M A D 4$ via homozygous deletion or mutation leads to decreasing SMAD4-dependent inhibition of transforming growth factor- $\beta$ (TGF- $\beta$ ) and the promotion of noncanonical TGF- $\beta$ signaling, thereby facilitating pro-tumorigenic responses. ${ }^{23,26,27}$ Recently, high-throughput sequencing studies have revealed genes with novel mutations/alterations with individual frequencies $<20 \%$, including lysine demethylase 6A (KDM6A) (18\%), rac family small GTPase 1 (RAC1) (10\%), ring-finger protein 43 (RNF43) (10\%), ATrich interaction domain $1 \mathrm{~A}(A R I D 1 A)(9 \%), \mathrm{B}$-Raf proto-oncogene, serine/threonine kinase (BRAF) (3\%), TGF- $\beta$ receptor 2 (TGFBR2) (3\%), mitogen-activated protein kinase kinase kinase 21 (MAP3K21) (3\%), switch/sucrose nonfermentable (SWI/SNF)-related, matrixassociated, actin-dependent regulator of chromatin, subfamily a, member 4 (SMARCA4) (3\%), activin A receptor type 2A (ACVR2A) (2\%), activin A receptor type 1B (ACVR1B) (2\%), N-ras protooncogene, GTPase (NRAS) (1\%), family with sequence similarity 133 member A $(F A M 133 A)(<1 \%)$, and zinc-finger matrin-type 2 (ZMAT2) $(<1 \%)^{24,28,29}$ It has been reported that $4-7 \%$ of pancreatic cancer patients have a germline DNA repair associated (BRCA) mutation. ${ }^{30}$ Furthermore, germline BRCA2 mutations have been observed in $5-17 \%$ of familial pancreatic cancer patients. ${ }^{31}$ Mutation of the GNAS complex locus (GNAS) at codon 201 has been observed exclusively in intraductal papillary mucinous neoplasms (IPMNs), a precursor to pancreatic cancer, at a rate of $41-75 \% .^{32}$ However, several studies have reported that $\sim 4 \%$ of pancreatic cancer patients have GNAS mutations. ${ }^{24}$ Most of these low-frequency mutations are in genes related to cellular processes such as cell survival, cell fate determination, and genome maintenance, involving various signaling pathways. ${ }^{28,33}$ In addition, recurrent noncoding mutations, which are enriched in transcriptionally active regions of the genome, also play an essential role in pancreatic cancer. ${ }^{34}$

Epigenetic alterations, which can regulate histone or DNA modification, play significant roles in molecular aspects of pancreatic tumorigenesis. ${ }^{35}$ In pancreatic cancer, epigenetic regulatory genes are frequently mutated, including those encoding SWI/SNF-mediated chromatin remodeling complexes and histone modification enzymes. ${ }^{36}$ Commonly mutated histone modification enzymes include the mixed-lineage leukemia (MLL) histone methylases (MLL2 and MLL3), histone methyltransferases, and the KDM6A histone demethylase. ${ }^{25,37}$ Furthermore, MLL2/3 and KDM6A exist in the same complex, which drives transcriptional activation through coordinated regulation of histone 3 lysine 4 (H3K4) methylation and histone 3 lysine 27 (H3K27) demethylation. ${ }^{38}$ During pancreatic cancer development, tumors with genetic defects in MLLs are more likely to induce expression of chromatin-regulating genes and cell proliferation-associated genes (including members of the SWI/SNF chromatin remodeling complex) as well as genes involved in cell cycle progression and cellular proliferation. ${ }^{37}$ The SWI/SNF complexes comprise multiple components encoded by different genes, including SWI/SNFrelated, matrix-associated, actin-dependent regulator of chromatin, subfamily a, member 2 (SMARCA2), SMARCA4, SMARCE1, $S M A R C B 1$, AT-rich interaction domain 2 (ARID2), ARID1A, AT-rich interaction domain $1 \mathrm{~B}(A R I D 1 B)$, and double PHD fingers 2 (DPF2). The overall prevalence of gene mutations or copy number variations in SWI/SNF complex components is $>10 \%$; thus, such alterations have important effects on chromatin remolding in pancreatic cancer pathogenesis. ${ }^{20,28,36}$ Histone deacetylases (HDACs), which catalyze histone deacetylation, are classified into two enzyme subtypes: the $\mathrm{NAD}^{+}$-dependent sirtuins (SIRT1-7) and the zinc-dependent HDAC1-11 proteins. $^{39}$ Interestingly, HDAC1 and HDAC2 are highly expressed in pancreatic cancer, and they can be recruited to the epithelial-cadherin $(\mathrm{CDH} 1)$ promoter (which is involved in histone deacetylation) by zinc-finger E-box binding homeobox 1 (ZEB1) or Snail, thereby promoting epithelialto-mesenchymal transition (EMT) and tumor metastasis. ${ }^{40,41}$ 4SC202 , a specific inhibitor of class I-HDACs, can inhibit TGF- $\beta$-induced EMT and p21 cyclin-dependent kinase inhibitor 1A (CDKN1A)mediated cell proliferation. ${ }^{42}$ SIRT6 ablation can potently cooperate with activated KRAS to promote pancreatic cancer metastasis via the hyperacetylation of histone 3 lysine 9 (H3K9) and histone 3 lysine 56 (H3K56) at the promoter of the lin-28 homolog B (LIN28b) gene, resulting in Myc recruitment and Lin28b upregulation. As a result, the expression levels of high-mobility group AT-hook 2 (HMGA2), insulin-like growth factor 2 mRNAbinding protein (IGF2BP) 1 , and IGF2BP3, which are downstream of target genes let- 7 , are increased. ${ }^{43}$

\section{Subtyping of pancreatic cancer}

Pancreatic cancer is a deadly malignancy that lacks effective therapeutics (Fig. 1b). This is in part due to the inter- and intratumor heterogeneity. ${ }^{44-46}$ Pancreatic cancer molecular subtype identification has the potential to improve clinical outcomes by allowing the development of individualized treatments. Recently, various molecular subtypes and subtypespecific treatment responses in pancreatic cancer have been recognized. ${ }^{36,44,47}$ The two predominant transcriptomic-based subtypes, which have been validated across multiple studies, are the classical/pancreatic progenitor subtype and the basal-like/ squamous/quasi-mesenchymal (QM-PDA) subtype. ${ }^{44,46,47}$ On the other hand, the existence of the exocrine-like/aberrantly differentiated endocrine exocrine (ADEX) subtype and the immunogenic subtype, which were proposed by Collisson et al. and Bailey and co-workers, have been controversial. ${ }^{44,47,48}$ Due to the desmoplastic nature of pancreatic cancer, low tumor cellularity remains a major concern in sampling and defining the molecular subtypes of this disease. Collisson et al. identified classical and QM-PDA subtypes within a large panel of pancreatic cancer cell lines, but did not detect an exocrine-like subtype. The Cancer Genome Atlas (TCGA) project found that the ADEX subtype and immunogenic subtype were associated with low cellularity, likely representing non-transformed cells. ${ }^{49}$ Later, Puleo et al. confirmed that the ADEX tumor subtype resulted from contamination of their pancreatic exocrine and endocrine components with adjacent normal pancreatic acinar cells. ${ }^{48}$ However, Noll et al. and Knudsen et al. identified the exocrine subtype in their patient-derived pancreatic cancer models. ${ }^{50,51}$ In addition, the exocrine subtype showed resistance to tyrosine kinase inhibitors and paclitaxel treatment via the expression of cytochrome P450 family 3 subfamily A member 5 (CYP3A5). ${ }^{50} \mathrm{~A}$ recent study reported significant variation in the tissue composition of pancreatic tumors from the Moffitt, Bailey, and TCGA cohorts. Up to $45 \%$ of the tumor samples from various public cohorts were reclassified after removal of the nonepithelial signal from the tumor bulk expression profiles. ${ }^{52}$

Signaling pathways regulating pancreatic cancer tumorigenesis and metastasis

Signaling pathways (e.g., RAS, phosphoinositide 3-kinase (PI3K)/ protein kinase $B(A K T)$, nuclear factor kappa-light-chain-enhancer of activated $B$ cells (NF-KB), janus kinase (JAK)/signal transducer and activator of transcription (STAT), Hippo/yes-kinase-associatedprotein (YAP), Wingless/int1 (WNT), etc.) have been linked to a variety of cancer-related cellular processes, including cell proliferation, differentiation, apoptosis, migration, angiogenesis, metabolism, and immune regulation. They have been implicated in pancreatic cancer tumorigenesis, prognosis, and resistance to therapy, it is likely that a deep understanding of these pathways will support the development of molecularly targeted pancreatic cancer therapies. 
RAS pathway. RAS signaling is centered on the activated small GTPase RAS, which drives the activation of three major effector pathways, RAS-like proto-oncogene A/B, RAF/MAP kinase (MEK)/ ERK, and PI3K. Oncogenic activation of RAS due to missense mutations is frequently detected in several types of cancer, including pancreatic cancer. ${ }^{53}$ Given that mutations in one RAS protein isoform, KRAS, are found in nearly $90 \%$ of pancreatic cancers, RAS signaling appears to play a critical role in both pancreatic cancer initiation and maintenance. Activated RAS can activate effector signaling pathways and transcription factors involved in cell transformation, proliferation, and metastasis. Activated RAS also promotes pro-inflammatory signaling via activation of NF-KB, signal transducer and STAT3, and glycogen synthase kinase-3/nuclear factor of activated T cell signaling. ${ }^{5-56}$ Pancreatic cancers without KRAS mutations show RAS activation via upstream signaling through receptor tyrosine kinases (RTKs), e.g., epidermal growth factor receptor (EGFR), and oncogenic activation of the downstream B-Raf proto-oncogene (B-RAF) molecule is detected in a small number of patients. ${ }^{57}$ Despite extensive insight into the molecular mechanisms of KRAS in multiple cancer-promoting effects, it has been challenging to obtain clinically effective KRAS inhibitors, with the exception of a KRAS G12C (carried by $\sim 1.5 \%$ of pancreatic cancer patients)selective inhibitor AMG $510 .^{58}$ In mouse models, KRAS inhibition can cause the activation of AKT, erb-b2 RTK2 (HER2), plateletderived growth factor receptor alpha, and EGFR, which might explain the inefficacy of these inhibitors. ${ }^{28}$

PI3K/AKT pathway. Since PI3K can be activated through an initial phosphorylation in response to oncogenic RAS, the PI3K/AKT pathway is frequently activated in human pancreatic cancer and mouse models of KRAS-driven pancreatic cancer. ${ }^{28,59}$ Abnormal overexpression or activation of AKT is associated with $>40 \%$ of pancreatic cancer patients. Abnormal AKT overexpression or activation was also reported to be associated with short survival and the pathologic grade of pancreatic cancer. ${ }^{60,61}$ It was demonstrated that PI3K/AKT activation appears to occur at the earliest stages of tumor evolution and that it controls pancreatic cell plasticity and carcinogenesis, as strong activation of $\mathrm{PI} 3 \mathrm{~K}$ signaling was observed in human acinar-ductal metaplasia (ADM) and in both low- and high-grade PanIN. ${ }^{62}$ Interestingly, PI3K p110a is required for pancreatic cell plasticity and cancer initiation induced by oncogenic KRAS, ${ }^{63}$ and constitutively active AKT1 cooperates with active KRAS (G12D) in the AKT pathway to accelerate pancreatic tumor onset and progression. ${ }^{64}$ Insulin-like growth factor (IGF) has been demonstrated to enhance the invasion and proliferation of pancreatic cancer cells via activation of the PI3K/AKT signaling pathway. ${ }^{65}$ Our recent study demonstrated that the PI3K/AKT/mTOR pathway regulates enolase 2 (ENO2) K394 deacetylation upon IGF-1 stimulation, thereby promoting liver metastasis in pancreatic cancer. ${ }^{66}$ Abnormal overexpression of IGF2BP2 caused by genomic amplification or posttranscriptional regulation promotes pancreatic cancer proliferation by activating the PI3K/AKT signaling pathway. ${ }^{67}$ In addition, multiple noncoding RNAs (ncRNAs), including miR-107, IncRNA ABHD11-AS1, IncRNA SNHG1, IncRNA AB209630, and transmembrane protein 158 have been reported to govern tumorigenesis, progression, metastasis, apoptosis, or drug resistance in pancreatic cancer cells via the PI3K/AKT pathway. ${ }^{68,69}$ Currently, several PI3K/AKT inhibitors are under investigation, but none are in routine clinical use. Some clinical trials of PI3K/AKT inhibitors (alone or in combination with chemotherapy drugs) in pancreatic cancer patients might reveal promising therapeutic effects.

NF-KB pathway. Constitutive activation of NF-KB, a transcription factor that is essential for inflammatory responses, has been frequently observed in pancreatic cancer. Increasing evidence suggests that both canonical and noncanonical NF-KB pathways can affect pancreatic cancer progression, metastasis, and drug resistance. ${ }^{7,71}$ KRAS mutation as well as oncogenic mutations in other genes, e.g., EGFR, PI3K, and TP53, also contribute to NF-KB activation in pancreatic cancer. ${ }^{72}$ Elevated levels of inflammatory cytokines and chemokines are observed in pancreatic cancer cells, and these increased levels are generally associated with the enhanced NF-KB signaling. ${ }^{73}$ For example, activated NF-KB translocates into the nucleus to enhance transcription of downstream inflammatory target genes, including interleukin-6 (IL-6), $\mathrm{IL}-8$, and IL-18, and increased levels of these cytokines lead to the activation of NF-KB signaling, thereby forming a positive feedback loop. ${ }^{7,75}$ Several studies have revealed that ncRNAs regulate the NF-KB signaling pathway by directly interacting with the functional domain or its transcripts. Overexpression of IncRNAPLACT1 promotes pancreatic cancer progression via constitutive activation of NF-KB signaling. MiR-628-5p functions as a tumorsuppressive microRNA (miRNA) in pancreatic cancer by negatively regulating phospholipid scramblase 1 and insulin receptor substrate 1 expression, which inhibits NF-KB signaling. ${ }^{76}$ Furthermore, NF-KB is reported to be involved in antitumor immunity. NF$K B$ in pancreatic stromal cells (PSCs) contributes to tumor growth by increasing the expression of $\mathrm{C}-\mathrm{X}-\mathrm{C}$ motif chemokine ligand 12 (CXCL12), which prevents cytotoxic $T$ cells from infiltrating the tumor and killing cancer cells. ${ }^{77} \mathrm{NF}-\mathrm{KB}$ is required for pancreatic cancer cells to evade macrophage surveillance, and growth differentiation factor 15-mediated inhibition of NF-KB signaling in infiltrating macrophages blocks the antitumor immune response during the early stages of tumorigenesis. ${ }^{78} \mathrm{~A}$ variety of NF-KB pathway inhibitors, including small molecules, peptides, small DNA/RNAs, viral proteins, and natural compounds, despite not being tested specifically in a pancreatic cancer model, have shown great promise. ${ }^{79}$

JAK/STAT pathway. The JAK/STAT pathway is clearly involved in many types of human cancer, including pancreatic cancer. High JAK2 expression predicts a poor prognosis in patients with PDAC. ${ }^{80}$ Several studies have shown that the JAK/STAT pathway is involved in inflammatory processes in pancreatic cancer. Both type 1 (i.e., IFNa and IFN $\beta$ ) and type 2 (i.e., IFN $\gamma$ ) interferons can upregulate programmed cell death 1-ligand 1 (PD-L1) expression via the JAK-STAT signaling pathway in pancreatic cancer. ${ }^{81,82}$ Sustained JAK/STAT pathway activation mediates chronic inflammation and impairs cytotoxic T lymphocyte (CTL) activation in pancreatic cancer, and the JAK-STAT inhibitor ruxolitinib can increase CTL infiltration to induce a Tc1/Th1 immune response in the tumor microenvironment of pancreatic cancer. ${ }^{83}$ In the PDAC microenvironment, it was demonstrated that tumor-derived IL-1 induces LIF expression and downstream JAK/STAT activation to generate inflammatory cancer-associated fibroblasts (iCAFs), whereas tumor-derived TGF- $\beta$ antagonizes this process by downregulating IL-1 receptor type 1 expression and promoting differentiation into myofibroblasts (myCAFs). ${ }^{84}$

Hippo/YAP pathway. YAP and its transcriptional coactivator with PDZ-binding motif (TAZ) are the two major downstream effectors of the Hippo pathway. ${ }^{85}$ Several studies have revealed that YAP is upregulated in pancreatic cancer patients, and YAP overexpression is correlated with liver metastasis and poor prognosis of pancreatic cancer. ${ }^{86}$ YAP has been reported to be insufficient to drive an initial step in the progression to pancreatic cancer with ADM. However, YAP is required for the induction of ADM progression to PanIN, and PanIN progression to pancreatic cancer. ${ }^{87,88}$ Accumulating studies have demonstrated that YAP is a critical player in pancreatic cancer progression in KRAS mutant mice. ${ }^{88}$ Active YAP promotes pancreatic tumor tumorigenesis, development, metastasis, stromal response, drug resistance, and metabolic homeostasis in KRAS-driven pancreatic cancer. ${ }^{89,90}$ 
However, it has been demonstrated that YAP is sufficient to drive PDAC recurrence in the absence of KRAS via bypass mechanisms involving YAP. ${ }^{91}$ Recent studies have also demonstrated that YAP is a major driver of the squamous subtype of pancreatic cancer, which is notably less dependent on oncogenic KRAS. ${ }^{92}$ These findings indicate that YAP not only acts as a pancreatic cancer driver downstream of KRAS but that it also substitutes for loss of oncogenic KRAS. ${ }^{93}$ Furthermore, YAP was identified as a critical regulator of the immunosuppressive microenvironment. YAP and TAZ can modulate the behavior of pancreatic stellate cells (PSCs) and influence the recruitment of tumor-associated macrophages (TAMs) and myeloid-derived suppressor cells. For example, YAP has been shown to promote the expression and secretion of multiple cytokines and chemokines, which in turn promote the differentiation and accumulation of myeloid-derived suppressor cells (MDSCs) in pancreatic cancer. ${ }^{94-96}$

WNT pathway. The WNT signaling pathway includes the canonical and noncanonical pathways and controls the maintenance of somatic stem cells in many tissues. Both the canonical ( $\beta$-catenin-dependent) and noncanonical ( $\beta$-catenin-independent) pathways have been implicated in pancreatic carcinogenesis, tumor progression, and therapeutic resistance. ${ }^{97,98}$ WNT signaling is required for KRAS-induced PanIN lesions and pancreatic cancer formation. ${ }^{99}$ KRAS activation can promote the invasion and migration of pancreatic cancer cells by regulating the WNT/ $\beta$-catenin signaling pathway or by increasing the interaction between $\beta$-catenin and cyclic AMP-response element-binding protein-binding protein. ${ }^{100}$ Increased WNT/ $\beta$-catenin signaling activation results in an enhanced stem cell-like phenotype of pancreatic cancer. ${ }^{101}$ Activation of the canonical pathway can prevent $\beta$-catenin degradation and promote its nuclear translocation, which enhances the transcription of targeted genes such as cyclin D1 and c-Myc. ${ }^{102,103}$ Aberrant nuclear accumulation of $\beta$-catenin is frequently found in PanIN and pancreatic cancer and is associated with their development. Canonical WNT ligands, such as Wnt family member 2 , Wnt family member $5 A$, and Wnt family member $7 \mathrm{~A}$, have been observed to be increased in pancreatic cancer tissues, and activated the WNT pathway, leading to the progression of pancreatic cancer. ${ }^{104,105}$ In addition, noncanonical ligands, such as mucin (MUC) family members (MUC1 and MCU4) and R-spondin, activate the WNT pathway, leading to pancreatic cancer progression. ${ }^{106-108}$ Pancreatic cancer is characterized by hypoxic conditions. Hypoxia-inducible factor-2a (HIF-2a) modulates WNT signaling by maintaining the levels of both SMAD4 and $\beta$-catenin during PanIN progression. ${ }^{109}$ Moreover, hypoxic conditions in pancreatic tumors stabilize HIF-2a, which interacts with $\beta$-catenin, leading to elevated canonical WNT/ $\beta$-catenin activity while favoring tumor progression. ${ }^{110}$

High invasion and metastasis of pancreatic cancer

Pancreatic cancer is generally considered an extremely aggressive tumor with high metastatic propensity. Most patients are diagnosed with advanced metastatic disease with a dismal prognosis. A better understanding of the mechanisms underlying metastatic progression is required to develop improved therapeutic interventions. ${ }^{111}$

It has been demonstrated that metastasis can occur during the early stages of pancreatic cancer, even before large mass formation by the primary tumor ${ }^{112,113}$ (Fig. 1c). It has been conceptualized that a stepwise accumulation of genetic and epigenetic alterations is the driving force for this process. ${ }^{114}$ Previous efforts to identify a signature of prometastatic genes or a consistent pattern of metastasis-driving mutations via genomic sequencing of human primary pancreatic tumors and their metastatic sites have failed. ${ }^{115}$ Nevertheless, distant metastatic lesions are hypothesized to evolve from the original tumor cells by acquiring distinct gene mutations. ${ }^{116}$
To metastasize, neoplastic cells must detach from the primary tumor and travel through the blood vessels or lymphatic system. A number of ncRNAs, ${ }^{117,118}$ transcription factors (e.g., Kruppellike factor 4 (KLF4), ${ }^{119} \mathrm{KLF} 5,{ }^{120} \mathrm{KLF}^{121}$ ), growth factors (e.g., vascular endothelial growth factor $(\mathrm{VEGF})^{122}$ ), and oxygen conditions $^{123}$ have been reported to drive pancreatic cancer metastasis. Using an organoid culture system, Roe et al. studied how transcription and the enhancer landscape evolves during the metastatic transition in a pancreatic cancer mouse model. They found that forkhead box A1-dependent enhancer reprogramming can promote the acquisition of metastatic traits. ${ }^{124}$ In pancreatic cancer, the most common metastatic site is the liver, followed by the lungs, peritoneum, and bones. ${ }^{125-127}$ Recently, Lee et al. reported that activation of STAT3 in hepatocytes and the subsequent production of serum amyloid $A 1$ and $A 2$ in the bloodstream direct pancreatic cancer liver metastasis. ${ }^{128}$ Interestingly, lineage-tracing analysis in a mouse model of pancreatic cancer revealed that metastases in the lung and liver are monoclonal, whereas those in the peritoneum and diaphragm tend to be polyclonal. These findings indicate that clonal diversity depends on the metastatic site. ${ }^{129}$ Global gene expression and molecular profiling studies showed that cytokines (e.g., IL-8), growth factors (e.g., hepatocyte growth factor and VEGF), and matrix metalloproteinase (e.g., extracellular matrix $(E C M)$ protease) ${ }^{122,130,131}$ as well as membrane receptors were key differentiating factors underlying pancreatic cancerderived hematogenous metastasis vs. peritoneal dissemination. $^{127}$ In pancreatic cancer, lymph node metastasis is considered a critical risk factor in patients with high-risk features. ${ }^{132}$ By using data and tumor samples from three independent cohorts, a recent study identified a miRNA signature associated with pancreatic cancer patients at risk for lymph node metastasis. ${ }^{133}$ Additional evidence has demonstrated that extracellular vesicles (EVs), such as exosomes, are essential for pancreatic cancer initiation and metastasis. ${ }^{134}$ Pancreatic cancer cell-derived exosomes can be taken up by Kupffer cells, resulting in TGF- $\beta$ secretion and upregulation of fibronectin production by hematopoietic stem progenitor cells, which then induce liver pre-metastatic niche formation. ${ }^{135}$ Moreover, exosome-derived integrin ( $\alpha 6 \beta 4, a 6 \beta 1$, and av $\beta 5$ ) uptake by resident cells (liver Kupffer cells, lung fibroblasts, or epithelial cells) contributes to organ-specific pre-metastatic niche formation via activation of SRC proto-oncogene ( $\mathrm{Src}$ ) phosphorylation and pro-inflammatory S100 gene expression. ${ }^{136}$ The extensively studied metastatic process of EMT, which represents the transition of epithelial cells into mesenchymal cells, has been shown to be pivotal during pancreatic cancer cell invasion and metastasis. ${ }^{137-139}$ TGF- $\beta$ was the first identified cytokine that induced EMT in pancreatic cancer cells via the RAS-MEK-ERK signaling pathway. ${ }^{140}$ Subsequent studies have demonstrated that EMT in pancreatic cancer cells can be triggered by various factors, including growth factors, cytokines, transcription factors, and miRNAs. ${ }^{141,142}$ Our group found that IGF-1 induced ENO2 deacetylation by HDAC3enhanced EMT, thus promoting liver metastasis of pancreatic cancer. ${ }^{66}$ In addition, pancreatic cancer cells undergoing EMT can induce a cancer stem cell (CSC) phenotype as well as drug resistance. ${ }^{143,144}$

The pancreatic tumor microenvironment is indispensable for pancreatic cancer progression. Reciprocal communication between cancer cells and stromal cells induces changes in cellular components of the pancreatic tumor microenvironment, which can prime the primary tumor for metastasis and cell migration. ${ }^{145}$ Primary pancreatic tumors can modulate the local microenvironment in metastatic sites by secreting exosomes and soluble factors to promote colonization. ${ }^{146,147}$ Various stromal cells, e.g., TAMs, also participate in angiogenesis and metastasis of pancreatic cancer via the secretion of VEGF, CXCL1, and CXCL8. ${ }^{148,149}$ 


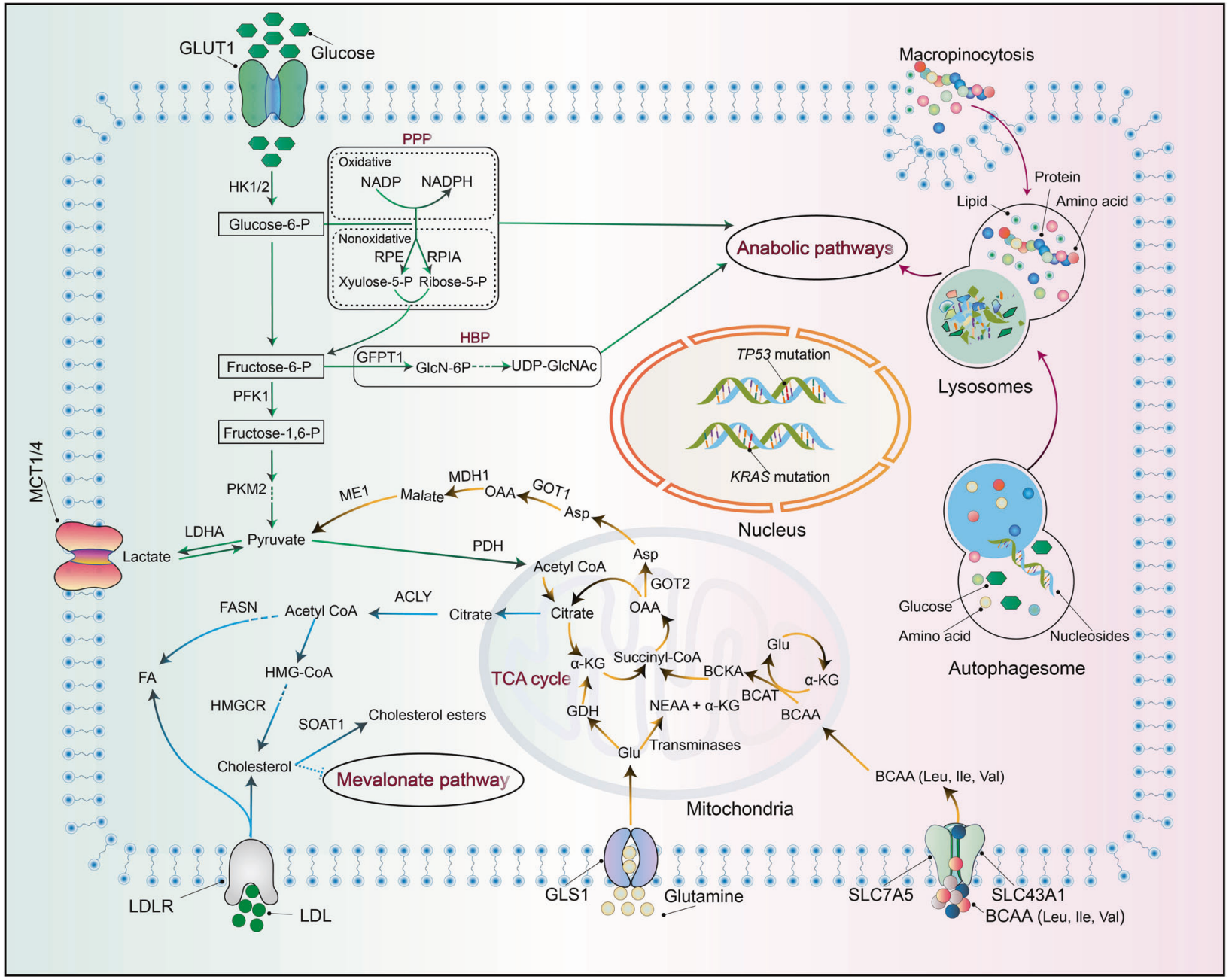

Fig. 2 Metabolic reprogramming in pancreatic cancer cells. KRAS activation and mutant TP53 enhance glucose metabolism to provide biosynthetic precursors for anabolic pathways, including the non-oxidative arm of the pentose phosphate pathway (PPP) and the hexosamine biosynthesis pathway (HBP). KRAS activation reprograms glutamine metabolism to sustain cellular redox homeostasis by increasing the $\mathrm{NADPH} / \mathrm{NADP}^{+}$ratio and recycling glutathione (GSH) via reduction of oxidized GSH. The BCAT2-mediated BCAA catabolism driven by KRAS plays a critical role during pancreatic cancer development. Enhanced nutrient salvaging, via the induction of macropinocytosis and autophagy, provides energy and regenerative nutrients, including glucose, amino acids, lipids, and nucleosides

\section{METABOLISM DYSREGULATION IN PANCREATIC CANCER}

Metabolic reprogramming is a hallmark of cancer, and it influences the survival and growth of cancer cells by providing energy and macromolecular precursors. ${ }^{150}$ Pancreatic cancer is characterized by hypovascularization, and pancreatic cancer cells are surrounded by a tight desmoplasia, thus creating a highly hypoxic and nutrient-limited microenvironment. ${ }^{151,152}$ Malignant pancreatic cancer cells proliferate uncontrollably and have a proclivity for distant metastasis, which results in increased demand for both energy and biosynthetic precursors (Fig. 1d). ${ }^{153}$ Metabolic reprogramming is central to the pathogenesis of pancreatic cancer driven by KRAS (Fig. 2). A recent study reported that pancreatic cancer tumors can be classified into four metabolic subgroups, i.e., quiescent, glycolytic, cholesterogenic, and mixed metabolic profiles, based on the expression levels of glycolytic and cholesterogenic genes. Glycolytic tumors correlate with poor outcomes in pancreatic cancer patients, whereas patients with cholesterogenic tumors tend to have better survival, possibly due to their higher energy expenditure. ${ }^{154}$

Reprogrammed glucose metabolism in pancreatic cancer Cancer cells can survive and proliferate in stressful microenvironments by reprogramming their energy metabolism to increase glucose uptake and enhance glycolysis and lactate production despite the presence of oxygen (known as the Warburg effect). ${ }^{155}$ Notably, pancreatic cancer cells exhibit extensive glucose metabolic reprogramming, including glycolytic enzyme overexpression and increased lactate production. ${ }^{152,156}$ It has been demonstrated that oncogenic KRAS promotes glycolysis by enhancing glucose uptake via upregulation of the glucose transporter (GLUT1) and several rate-limiting glycolytic enzymes, including hexokinase 1 and 2, phosphofructokinase-1, and lactate dehydrogenase A. ${ }^{156,157}$ Furthermore, in pancreatic cancer cells, mutant KRAS signaling promotes mitochondrial translocation of phosphoglycerate kinase-1, resulting in the production of phosphorylated pyruvate dehydrogenase kinase- 1 and restricted oxidative phosphorylation (OXPHOS). ${ }^{158}$ Enhanced glycolysis induced by oncogenic KRAS provides biosynthetic precursors for anabolic pathways, including the hexosamine biosynthesis pathway (HBP) and the nonoxidative arm of the pentose phosphate pathway (PPP). ${ }^{156} \mathrm{KRAS}$ mutations drive the HBP, a side path of glycolysis, to increase the generation of biosynthetic precursors required for protein glycosylation and the synthesis of proteoglycans, glycolipids, and glycosyl phosphatidylinositol anchors. ${ }^{156,159}$ This process depends on both increased glycolysis and transcriptional upregulation of glutamine $(\mathrm{Gln})$ fructose 6-phosphate transamidase 1 
in the HBP. ${ }^{156}$ Similarly, oncogenic KRAS enhances the flux of glucose-derived carbon into the non-oxidative arm of the PPP to generate ribose-5-phosphate for de novo nucleotide biosynthesis, thus fueling proliferation. ${ }^{160}$ In addition, p53 also plays a vital role in metabolic reprogramming in pancreatic cancer cells. It has been demonstrated that KRAS and p53 can promote metabolic changes even before the occurrence of malignant transformation. ${ }^{161}$ In pancreatic cancer cells, mutant TP53 can activate GLUT1-mediated glucose transport by upregulating paraoxonase 2 expression and impairing the expression of TP53-induced glycolysis regulatory phosphatase to promote glycolysis. ${ }^{162}$ A gain-of-function TP53 allele was demonstrated to enhance the Warburg effect by inducing GLUT1 translocation to the plasma membrane, which is regulated by the RAS homolog family member A/rho-associated coiled-coil containing protein kinase/GLUT1 signaling pathway. ${ }^{163}$ In addition, TP53 mutations can decrease mitochondrial activity to suppress pancreatic cancer progression. ${ }^{164}$ Highly hypoxic microenvironments commonly form during pancreatic cancer progression, thus increasing the expression and stability of HIF-1. The hypoxic condition in the tumor microenvironment and HIF-1a upregulation contributes to elevated glycolytic activity and these factors cooperate with mutant KRAS to sustain cytosolic ATP generation even though knockdown of its expression has limited influence on metabolism-related enzyme expression. ${ }^{156,165}$ HIF-1a upregulation has been shown to upregulate GLUT1 as well as the expression levels of glycolysis-related genes to sustain cytosolic ATP levels in pancreatic cancer cells. ${ }^{166,167}$ HIF-1a also upregulates the expression of GFPT2 and inhibits the expression of pyruvate dehydrogenase to restrict mitochondrial oxidation. ${ }^{168}$

Reprogrammed amino acid metabolism in pancreatic cancer It has been demonstrated that amino acid metabolism plays a crucial role in pancreatic cancer progression. ${ }^{169,170}$ Among the amino acids, GIn is the most abundant in circulation and is a major source of carbon and nitrogen for cancer cells. ${ }^{171}$ Notably, Gln is essential to support pancreatic cancer growth via redox homeostasis in a KRAS-driven metabolic pathway. ${ }^{156,172,173}$ Inhibition of downstream components of Gln metabolism leads to decreased tumor growth. ${ }^{151,172}$ Modification of the activity of glutaminase (GLS), which catalyzes the first step in glutaminolysis, i.e., the conversion of $\mathrm{Gln}$ to glutamate $(\mathrm{Glu})$ in mitochondria, represents a viable therapeutic strategy. Interestingly, pancreatic cancer cells exhibit compensatory metabolic networks that sustain progression after GLS inhibition. ${ }^{174}$ When GLS1 was inhibited in pancreatic tumors, the GLS2 pathway was upregulated for Glu production. ${ }^{175}$

Arg can be directly catalyzed by oxide synthases (NOS1-3) into citrulline and nitric oxide (NO). NO promotes pancreatic cancer cell proliferation, and this effect can be dampened by knocking down NOS3 activity in KRAS mutant tumors. ${ }^{176}$ Arg deprivation is currently being evaluated for safety and efficacy in clinical trials. A recent study reported that Arg deprivation inhibits pancreatic cancer cell migration, invasion, and EMT. ${ }^{177}$ The mammalian target of rapamycin complex 1 (mTORC1) kinase is a master growth regulator that senses many environmental cues, including amino acid levels. ${ }^{178,179}$ Via solute carrier family 38 member 9 , Arg serves as a lysosomal messenger that couples mTORC1 activation to the lysosomal release of essential amino acids needed to drive pancreatic cancer cell growth. ${ }^{179,180}$

In addition, proline dehydrogenase (PRODH)/proline oxidase, which catalyzes the first step of proline degradation, has been extensively linked to the progression of cancer, including pancreatic cancer. ${ }^{181}$ PRODH1 expression is elevated in pancreatic cancer cells to sustain cellular survival and proliferation under lowglucose or Gln-limited conditions. ${ }^{182}$ Via experiments with genetically engineered mouse models (GEMMs) and primary pancreatic epithelial cells, a recent study demonstrated that induction of the serine-glycine one-carbon pathway plays a vital role in tumorigenesis. ${ }^{183}$ In recent years, branched-chain amino acid (BCAA) metabolism has been tentatively linked to pancreatic cancer development. ${ }^{184}$ As essential amino acids, BCAA must be derived from the extracellular milieu, and specific transporters responsible for BCAA (isoleucine, leucine, and valine) absorbance include members of the solute carrier family 7 (SLC7) family (SLC7A5A and SLC7A8) and the solute carrier family 43 (SLC43) family (SLC43A1 and SLC43A2). ${ }^{185,186}$ It has been reported that plasma BCAAs are elevated in patients with increased pancreatic cancer risk and in mice with early-stage pancreatic cancer driven by mutant $K R A S^{187}$ BCAA transaminase 2 (BCAT2), a BCAA transaminase, is upregulated in advanced pancreatic cancer. ${ }^{188}$ A recent study demonstrated that BCAT2 plays a central role in the development of ductal-derived PanIN lesions by sustaining BCAA catabolism, and BCAT2-mediated BCAA catabolism driven by KRAS is critical for pancreatic cancer development

Reprogrammed lipid metabolism in pancreatic cancer

Lipid metabolism is an essential cellular process that converts nutrients into metabolic intermediates for the maintenance of cellular structures, energy storage, and the generation of signaling molecules production. It is increasingly reported that dysregulation of lipid metabolism is associated with the progression of various cancers, including pancreatic cancer. ${ }^{189,190}$

In mice, a high-fat diet can increase oncogenic KRAS activity, leading to fibrosis, inflammation, and enhanced PDAC development. ${ }^{191}$ It has been demonstrated that multiple enzymes that catalyze de novo fatty acid (FAs) synthesis, including ATP citrate lyase, FA synthase (FASN), and stearoyl-CoA desaturase, are obviously upregulated in pancreatic cancer. ${ }^{192,193}$ In addition, increased FASN expression was correlated with poor survival and poor gemcitabine responsiveness through enhanced estrogen receptor stress and cancer stemness. Inhibition of FA biosynthesis could be a promising strategy to overcome gemcitabine resistance in pancreatic cancer. ${ }^{194}$ Interestingly, different FAs might play different roles in pancreatic cancer. Saturated FAs promote tumor progression by increasing cyclooxygenase-2, VEGF, and caveolin 1 expression and the production of lipid droplets (LDs). High-fat diets enriched in monounsaturated FAs and $\omega 6$ polyunsaturated FAs (PUFAs) can increase tumor size in pancreatic cancer mouse models, and $\omega 6$ PUFA-rich diets frequently lead to liver metastasis by increasing hepatic $\omega 6$ PUFAs levels. ${ }^{195} \omega 3$ PUFAs decrease pancreatic cancer cell proliferation by reducing AKT phosphorylation, while $\omega 6$ PUFAs can promote tumor growth by increasing AKT phosphorylation. ${ }^{196}$ FA $\beta$-oxidation (FAO) is essential for energy maintenance to support the proliferation and survival of cancer cells. ${ }^{197,198}$ A recent study demonstrated that the main source of ATP production in pancreatic cancer depends on FAO rather than glycolysis. ${ }^{199}$ Excess lipids stored as LDs can be converted into FAs by adipose triglyceride lipase and hormone-sensitive lipase (HSL) to provide ATP for pancreatic cancer metastasis. A recent study demonstrated that oncogenic mutant KRAS in pancreatic cancer facilitated LD utilization via suppressing the expression and phosphorylation of HSL, thereby fueling cancer cell invasion. ${ }^{200,201}$

In an oncogenic KRAS mouse model, the comparative transcriptomic analysis identified lipid-related metabolic pathways, in particular cholesterol uptake, as being the most highly enriched in pancreatic cancer (compared with a normal pancreas). ${ }^{192}$ Pancreatic cancer patients with increased expression of cholesterol synthesis genes showed a survival benefit in resectable and metastatic cases. ${ }^{154}$ Furthermore, cholesterol metabolism plays an important role in controlling pancreatic cancer development and differentiation. By using a GEMM driven by KRAS ${ }^{\mathrm{G} 12 \mathrm{D}}$ mutation and homozygous TP53 loss, a recent study demonstrated that inhibition of cholesterol biosynthesis with statins, a widely used inhibitor of the mevalonate pathway, or NAD (P)-dependent steroid dehydrogenase-like enzyme inhibitor can 
switch glandular pancreatic carcinomas to a basal phenotype. ${ }^{202}$ Loss of function of TP53 can drive tumorigenesis via downregulation of cholesterol transporter gene ATP-binding cassette subfamily A member 1 and activation of $\mathrm{PI} 3 \mathrm{~K} /$ sterol regulatory element-binding protein 2 (SREBP2) maturation. Consequently, the mevalonate pathway, which is responsible for cholesterol and sterol biosynthesis, is upregulated. ${ }^{203,204}$ Statins can modestly affect cholesterol homeostasis and significantly reduce the synthesis of electron carrier coenzyme $Q$, leading to severe oxidative stress and apoptosis in pancreatic cancer. ${ }^{205}$ By using organoid and mouse models, a recent study demonstrated that the loss of sterol O-acyltransferase 1 (SOAT1), a key enzyme catalyzing cholesterol to inert cholesterol esters to sustain mevalonate pathway flux, significantly impairs pancreatic cancer progression. Mechanistically, mutant TP53 and TP53 LOH can promote the expression of SOAT1, which upholds SREBP2-driven cholesterol biosynthesis to sustain the levels of multiple nonsterol isoprenoids, e.g., farnesyl pyrophosphate and geranylgeranyl pyrophosphate, ultimately resulting in RAS and Rho activation. These findings indicate that SOAT1 inhibition might be a potential therapeutic modality for pancreatic cancer patients with mutant TP53 and TP53 $\mathrm{LOH}^{206}$

Autophagy and nutrient salvage regulation in pancreatic cancer Autophagy, a cellular "self-eating" process, allows cells to degrade and recycle their own cellular components, thereby enhancing their survival under stress conditions. Notably, it seems that autophagy has conflicting functions during cancer progression, including both tumor-suppressive and tumor-promoting effects, which are likely context-dependent. The role of autophagy in pancreatic cancer is also complex. During early stages, autophagy is antitumorigenic because of its function in cellular quality control, while in established cancer, autophagy can support cancer cell survival and progression by providing resources for macromolecule biosynthesis and bioenergetics. ${ }^{207} \mathrm{~A}$ growing body of evidence suggests that autophagy is required for pancreatic cancer cell survival and metabolism. Genetic depletion of autophagic components or pharmacological inhibition of autophagy with chloroquine can decrease tumorigenicity in vivo. ${ }^{208,209}$ Oncogenic KRAS can activate autophagy, and knockdown of KRAS expression or inhibition of its downstream MEK/ERK signaling cascade further induces autophagy and decreases both glycolytic and mitochondrial functions. Combined autophagy inhibition by hydroxychloroquine (HCQ) and MEK/ERK inhibitors showed enhanced antitumor activity in vivo. ${ }^{209,210}$ Although autophagy is usually regulated by nutrient and oxygen availability, pancreatic cancer cells often display high basal levels of autophagic flux even under nutrient-rich conditions. ${ }^{211}$ Pancreatic cancer cells use autophagy to obtain glucose and amino acids from catabolized substrates to fuel the citric acid cycle (TCA) cycle, OXPHOS, and ATP biosynthesis. It has been shown that autophagy induction in pancreatic cancer cells occurs as part of a broader transcriptional program regulated by the microphthalmia/transcription factor $E$ (MiT/TFE) family of transcription factors. ${ }^{212}$ MiT/TFE-dependent autophagy-lysosome activation is required to maintain intracellular amino acid pools, and knockdown of the encoding genes strongly suppresses tumor progression. $^{212}$ In vitro, autophagy inhibition leads to disrupted redox state, elevated DNA damage, and decreased levels of metabolic substrates, resulting in suppression of pancreatic cancer cell proliferation. ${ }^{209,213}$ Notably, the disrupted redox state and defective mitochondrial respiration caused by autophagy inhibition can be rescued by supplying antioxidants and TCA cycle intermediates. ${ }^{213,214}$ Autophagy is also a key regulator of immunogenicity in pancreatic cancer cells by virtue of its selective targeting of major histocompatibility complex class I (MHC-I) for degradation. Elevated autophagy is strongly correlated with increased immune evasion and reduced $\mathrm{CD}^{+} \mathrm{T}$ cell infiltration in pancreatic cancer. $^{215,216}$

\section{THE TUMOR MICROENVIRONMENT OF PANCREATIC CANCER}

The tumor microenvironment in pancreatic cancer is highly immunosuppressive and is characterized by an abundant stromal response as a desmoplastic reaction. Accumulating research has highlighted the critical roles of the tumor microenvironment in maintaining pancreatic cancer development; hence, the tumor microenvironment acts as a key determinant by which this malignancy acquires therapeutic resistance to currently available treatment.

The pancreatic stroma in pancreatic cancer

Pancreatic cancer is characterized by an extensive and dense fibrous stroma, and the stromal composition can account for up to $90 \%$ of the total tumor volume. The crosstalk between tumor cells and the stromal microenvironment is complex, and stromal elements regulate pancreatic cancer progression in a more complex manner. The paradoxical effects of the pancreatic cancer-associated stroma on pancreatic cancer cells, which include both tumor-promoting and tumor-suppressive regulations, might represent a target for new therapeutic strategies based on context-dependent stromal alterations. ${ }^{217,218}$

The composition of the pancreatic cancer stroma. Pancreatic cancer is known for its stroma/desmoplastic reaction, comprising a heterogeneous mass of cells, including PSCs, fibroblasts, immune cells, ECM, and soluble proteins such as cytokines and growth factors. ${ }^{219}$ The main types of stromal cells that contribute to tumor progression are PSCs, CAFs, TAMs, regulatory $\mathrm{T}$ cells (Tregs), and MDSCs. ${ }^{219}$ PSCs can be classified as quiescent PSCs (qPSCs) or activated PSCs (aPSCs) depending on their activation state. As lipid storage cells in the pancreas, qPSCs are located in surrounding perivascular regions or basolateral aspects of acinar cells. ${ }^{20}$ qPSCs can be activated under some environmental stress conditions, including inflammation, hypoperfusion, and oxidative stress, etc. aPSCs can transdifferentiate into myofibroblast-like phenotype, constituting a growth-permissive microenvironment in the tumor tissue. ${ }^{221}$ However, aPSCs are not permanently sustained because they can revert to their inactivate state under the influence of several factors, e.g., apoptosis, senescence, tissue regression, or recovery. ${ }^{220}$ Macrophages, a key class of innate immune cells, are derived from the mononuclear phagocyte system in the bone marrow, and they contribute to desmoplasia and immunosuppression of pancreatic cancer. TAMs can respond to environmental signals (e.g., inflammatory cytokines IL-8, IL-6, IL-1 $\beta, \mathrm{IL}-10),{ }^{222}$ and it is thought that a high number of infiltrating TAMs may correlate with tumor size, prognosis, and patient survival in pancreatic cancer. $^{223}$ Forkhead box P3-positive $\left(\right.$ Foxp $\left.^{+}\right)$Tregs are a subtype of $T$ cells with significant roles in immunological selftolerance maintenance and immunosuppression modulation during tumor progression. Foxp ${ }^{+}$Tregs have been shown to interact with tumor-associated $\mathrm{CD}_{11} \mathrm{c}^{+}$dendritic cells and to restrain the immunogenic activation of $\mathrm{CD}^{+} \mathrm{T}$ cells. ${ }^{224}$ In pancreatic cancer, Foxp3 ${ }^{+}$Tregs are an essential source of TGF- $\beta$ ligands, promoting the differentiation of $\mathrm{SMA}^{+}$fibroblasts (myCAF) for tumor progression. Furthermore, Foxp ${ }^{+}$Treg depletion results in the differentiation of inflammatory fibroblast subsets, which in turn drive increased myeloid cells recruitment and impair the alleviation of immunosuppression. ${ }^{225}$

MDSCs are a heterogeneous population of immature myeloid cells that can be found in both tumors and the spleen. MDSCs usually participate in various pathological processes, including tissue inflammation and tumor progression. MDSCs can build crosstalk with TAMs, Tregs, and other immune cells to suppress effector $T$ cells in the tumor microenvironment. ${ }^{226}$ Pancreatic tumors can create a hypoxic environment that recruits the MDSCs to the tumor microenvironment where they exert their immunosuppressive effect. $^{227}$ 
The origin and functions of CAFs in pancreatic cancer. CAFs are one of the most prominent and active components of the desmoplastic stroma of pancreatic cancer. They possess diverse functions, including a robust fibro-inflammatory stromal effect and extensive reciprocal crosstalk with pancreatic cancer cells. ${ }^{228}$ The exact origin of CAFs is unclear at this time because they are derived from various types of cells, e.g., adipocytes, epithelial cells, resident fibroblasts, and bone marrow-derived mesenchymal stem cells. $^{221}$ The general consensus is that aPSCs are one of the predominant origins of CAFs in the pancreatic cancer stroma. ${ }^{229}$ Moreover, CAFs activation involves multiple complex pathways and cytokines, including EMT, the sonic hedgehog $(\mathrm{SHH})$ pathway, and various cytokines, including IL-1, IL-6, IL-10, TGF- $\beta$, and tumor necrosis factor (TNF-a). ${ }^{228,230}$

As major players in pancreatic tumorigenesis, activated CAFs from PSCs have numerous anti- and pro-tumorigenic influences on cancer cells in the tumor microenvironment. CAFs establish signal communication with tumor cells through a variety of mechanisms, e.g., EVs and classical paracrine. ${ }^{221}$ CAFs can increase the pancreatic cancer aggressiveness via EVs crosstalk that carries the annexin A6 (ANXA6)/LDL receptor-related protein 1/thrombospondin 1 complex under physiopathologic conditions (macrophages invasion, hypoxia, and lipid starvation). ${ }^{231}$ The presence of $\mathrm{ANXA6}^{+}$EVs in the peripheral blood is restricted to pancreatic cancer patients, establishing these EVs as a potential biomarker of pancreatic cancer aggressiveness. ${ }^{231}$ Moreover, CAF-derived EVs can inhibit the metabolic reaction of mitochondrial OXPHOS and increase glycolysis and Gln-dependent reductive carboxylation in pancreatic cancer cells. ${ }^{232}$ Under chemotherapy, exposure of pancreatic cancer to gemcitabine might increase the release of CAF-derived EVs, which might further enhance chemoresistance and tumor cell survival. ${ }^{233}$ High expression of fibroblast activation protein (FAP) in CAFs has been established as a key enhancer of PDAC progression. Genetic deletion of FAP in the KPC mouse model results in delayed primary tumor formation in mice bearing pancreatic cancer tumors, leading to improved survival. Currently, researchers are attempting to establish FAP as a precise molecular target for guiding pancreatic cancer therapy. ${ }^{234}$ CAFs can enhance aerobic glycolysis and secrete high-energy metabolites, including lactate and pyruvate. ${ }^{235}$ Moreover, it has been demonstrated that enhanced autophagy in CAFs contributes to the release of amino acids, which tumor cells can use as anabolic substrates. ${ }^{236}$ CAFs also supply amino acids, TCA cycle intermediates, and lipids to tumor cells via exosomes, thus promoting pancreatic tumor growth, especially under nutrient-limited conditions. ${ }^{232}$ Lysophosphatidylcholine released from CAFs can be converted into lysophosphatidic acid (LPA) by autotaxin within the tumor microenvironment, subsequently exerting mitogenic signals via binding with the LPA-receptor on the surface of tumor cells, to support tumor growth. ${ }^{237}$

CAF heterogeneity in pancreatic cancer. Due to differences in their sources of origination and activation mechanisms, CAFs display heterogeneity that is characterized by several subgroups with different phenotypes and functions in pancreatic cancer tissue. For a long time, CAFs were only considered tumor promoters in the tumor microenvironment of the pancreatic cancer cells based on their involvement in tumor cell proliferation and survival via facilitating tumor invasion, metastasis, and immunosuppression. ${ }^{238,239}$ For example, as one of the traditional CAF activation pathways, the $\mathrm{SHH}$ pathway has been targeted by genetic deletion or chemical inhibition for tumor suppression. However, some recent studies have found that tumors can become more aggressive and undifferentiated upon Shh deletion in a pancreatic cancer mouse model. ${ }^{218,240}$ This evidence suggests that CAFs might comprise several subsets that have different or even opposing effects on tumor progression. Biffi et al. have identified two CAFs subtypes with either myofibroblastic characteristics or inflammatory phenotypes, which they have named "myofibroblastic CAFs" and "inflammatory CAFs," respectively. Despite these observations, the exact origins of CAFs and their roles in pancreatic cancer progression have not yet been fully explored. ${ }^{84}$ It has been reported that genetically regulated phenotypes of pancreatic cancer affect pancreatic cancer progression by increasing matricellular fibrosis and tissue tension. ${ }^{241}$ Tape et al. found that oncogenic KRAS ${ }^{G 12 D}$ engages heterotypic fibroblasts, which subsequently instigate reciprocal signaling in tumor cells. ${ }^{242}$

Classical therapeutic approaches that focus on the depletion of the stromal desmoplasia usually lead to a disappointing result. In light of the complexity of the pancreatic cancer microenvironment, ${ }^{243}$ future therapeutic approaches that target both the tumor and the stroma may be able to achieve a better outcome.

\section{Immunomodulation in pancreatic cancer}

Pancreatic cancer is often considered to be immunosuppressive based on a massive infiltration of immunosuppressive leukocytes and minimal antitumor T cell infiltration. ${ }^{244}$ Pancreatic cancer cells have been shown to cooperate with other regulatory immune cells to evade immune surveillance and to resist the cytotoxic effect of T lymphocytes (Fig. 3). ${ }^{245}$ Previous studies have shown that there is a correlation between the clinical significance and the composition and quantity of tumor-infiltrating immune cells and tumor-associated myofibroblasts. ${ }^{246}$ Mahajan et al. described a novel prognostic signature comprising distinct immune cell and stromal components for risk assessment of pancreatic cancer patients and prediction of their progression-free survival (PFS). ${ }^{247}$ Research has also shown that patients with higher levels of CD4 ${ }^{+}$ and/or $\mathrm{CD}^{+} \mathrm{T}$ cells have significantly longer survival. ${ }^{248}$

The immunosuppressive tumor microenvironment of pancreatic cancer can be largely attributed to tumor cell-intrinsic pathways. Oncogenic KRAS mutations and KRAS-induced colony-stimulating factor (CSF) 2 (GM-CSF) production, promote pancreatic neoplasia progression via an influx of $\mathrm{CD} 11 \mathrm{~b}^{+} \mathrm{Gr} 1^{+}$immunosuppressive cells and suppressed antitumor T cell function. ${ }^{249}$ PISK is a critical downstream effector of KRAS. One of its catalytic subunits, phosphatidylinositol-4,5-bisphosphate 3-kinase catalytic subunit alpha, has been shown to reduce the expression of MHC class I and CD80 in pancreatic cancer cells, leading to limited T cell recognition and pancreatic cancer cell clearance. ${ }^{250}$ Moreover, p53 and KRAS can cooperatively target the ADP ribosylation factor 6ArfGAP with SH3 domain, and ankyrin repeat and $\mathrm{PH}$ domain 1 pathway to promote pancreatic cancer immune evasion, PD-L1 dynamics, and malignancy. ${ }^{251}$ Loss of p53 function activates JAK2STAT3 signaling, which induces macrophage and neutrophil infiltration, reduces $\mathrm{CD}^{+} \mathrm{T}$ cell levels, and promotes tumor growth in pancreatic tumors in mice. ${ }^{252}$ In neoplastic pancreatic cancer cells, focal adhesion kinase (FAK) activity is hyperactivated and is correlated with high levels of TAMs, MDSCs, and Treg cells and low $\mathrm{CD}^{+}$cytotoxic T cell infiltration. ${ }^{253,254}$ In addition, FAK inhibition increases immune surveillance by mitigating the immunosuppressive pancreatic cancer tumor microenvironment, thereby rendering tumors responsive to immunotherapy. ${ }^{254}$ Similarly, inhibition of endogenous Myc triggers ubiquitous regression of pancreatic tumors by decreasing the levels of neutrophil and macrophage infiltration. ${ }^{255}$ Studies in a pancreatic cancer mouse model have demonstrated that Myc activation alone is sufficient to trigger the release of instructive signals that cooperatively coordinate changes in multiple immune and stromal cell types. ${ }^{256}$ Moreover, Myc and KRAS ${ }^{\mathrm{G} 12 \mathrm{D}}$ cooperatively regulate gene expression resulting in natural killer cell-mediated immune surveillance. ${ }^{257}$

Aside from genetic alterations that regulate the tumor microenvironment, pancreatic cancer can adopt other strategies of immune evasion, including the production of metabolites and immunosuppressing chemokines and cytokines. Lactate is robustly exported from pancreatic cancer cells via elevated 


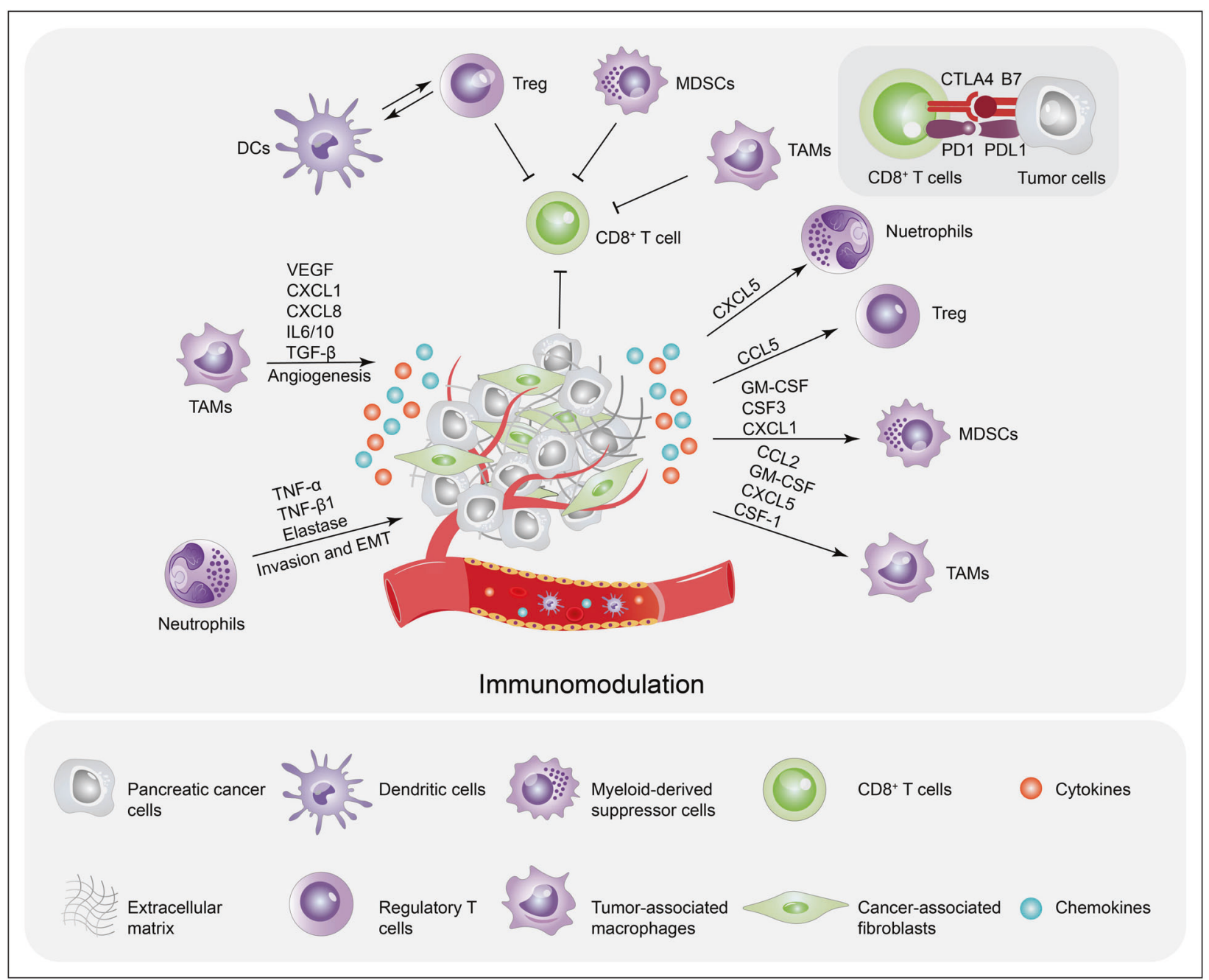

Fig. 3 Immune evasion orchestrated by pancreatic cancer cells and stromal cells. The secretion and immunomodulation of pro-tumorigenic cytokines by pancreatic cancer cells and stromal cells are tightly regulated by oncogenic KRAS- or mutant TP53-dependent pathways. Pancreatic cancer cells secrete cytokines and, chemokines and recruit immunosuppressive cells, including MDSCs, TAMs, Treg cells, and neutrophils, which suppress the activity and functions of $\mathrm{CD}^{+}$cytotoxic T cells. Pancreatic cancer cells also evade the immune system by expressing PDL- 1 to promote $\mathrm{CD}^{+}{ }^{\mathrm{T}}$ cell exhaustion. Immune cell infiltration also releases cytokines and growth factors that directly stimulate tumor growth by promoting angiogenesis and increasing the invasive ability of pancreatic cancer cells

monocarboxylate transporter 4 (MCT4)4 expression, and neighboring tumor cells in normoxic regions overexpress the lactate importer MCT1 to fuel OXPHOS and promote tumor growth. ${ }^{168,258}$ Furthermore, lactate secreted from pancreatic cancer cells remodels the tumor microenvironment and contributes to the M2-like polarization of TAMs, which leads to suppressed immunity. ${ }^{259,260}$ TAMs participate in metabolic crosstalk and elevate the glycolytic signature, which in turn promotes pancreatic cancer vascularization and metastasis. ${ }^{261}$ TAM-secreted C-C motif chemokine ligand 18 (CCL18) interacts with ITPNM family member 3 to induce vascular cell adhesion molecule- 1 overexpression in pancreatic cancer cells, thereby promoting lactate production accompanied by elevated aerobic glycolysis. This effect activates macrophages into a TAM-like phenotype, forming a positive feedback loop. ${ }^{259}$ Pancreatic cancer cells can secrete CCL5 to promote the recruitment of Treg cells into the tumor site. $^{262,263}$ The levels of tumor-derived GM-CSF, ${ }^{249}$ M-CSF, ${ }^{249}$ CSF1, ${ }^{264}$ CCL2, and CXCL5 ${ }^{265,266}$ are often highly elevated by mutant KRAS expression and are associated with TAM infiltration. The chemokine $\mathrm{CCL} 2$, which can be produced by pancreatic cancer cells, facilitates the recruitment of immunosuppressive $\mathrm{C}-\mathrm{C}$ motif chemokine receptor 2-positive TAMs into the tumor microenvironment, thereby limiting T cell infiltration. ${ }^{267,268}$ CXCL5 is an important attractant for immune cell accumulation. Pancreatic cancer patients with high CXCL5 expression have more intratumoral M2-polarized macrophages, neutrophils, and $\mathrm{lgG}^{+}$ plasma cells than those with low CXCL5 expression. ${ }^{269}$ Studies have demonstrated that tumor-derived CXCL1, ${ }^{270} \mathrm{GM}-\mathrm{CSF}^{2}{ }^{271}$ and CSF $-3^{272}$ mediate the recruitment of MDSCs to the tumor microenvironment. CXCL1 has been shown to facilitate the recruitment of myeloid cells and inhibition of the T cell infiltration into tumors. ${ }^{273}$ Moreover, CSF1 serum levels are upregulated in pancreatic cancer, and higher levels correlate with increased macrophage infiltration and advanced tumor stage. ${ }^{274,275}$ Pancreatic cancer cells also secrete various immunosuppressive cytokines, including TGF- $\beta$ and IL-10, which (1) coordinate immunosuppressive tumor microenvironment formation and (2) recruit cells involved in immune evasion, e.g., TAMs and Treg cells, to evade antitumor immunity. ${ }^{276,277}$ Foxp3 expressed by pancreatic cancer cells can activate CCL5 to promote the recruitment of Treg cells from peripheral blood to the tumor site, which negatively correlates with poor prognosis in pancreatic cancer. ${ }^{262}$ It has been demonstrated that neutrophils can increase pancreatic cancer metastasis by releasing a collection of cytokines, including 
TNF- $\alpha$, TGF- $\beta 1$, and elastase. ${ }^{278,279}$ Moreover, increased PD-L1 expression on pancreatic cancer cells can restrain $T$ cell function by promoting $\mathrm{CD} 8^{+} \mathrm{T}$ cell exhaustion. Pancreatic cancer can even induce $\mathrm{CD}^{+} \mathrm{T}$ cell apoptosis via a Fas/FasL counterattack. ${ }^{280,281}$

\section{CLINICAL PERSPECTIVES OF CURRENT ADVANCES IN PANCREATIC CANCER}

Novel biomarkers of pancreatic cancer based on liquid biopsy Currently, pancreatic cancer detection and diagnosis primarily rely on imaging modalities, including transabdominal ultrasonography, computed tomography (CT), magnetic resonance imaging), positron emission tomography, endoscopic retrograde cholangiopancreatography, and endoscopic ultrasonography. ${ }^{282}$ These screening technologies have limitations in their detection capability. For instance, they are ineffective at detecting earlystage pancreatic cancer and small metastases or peritoneal lesions, etc. ${ }^{283,284}$ Serum tumor markers such as carcinoembryonic antigen and carbohydrate antigen have been widely used for pancreatic cancer diagnosis in clinical settings. However, they lack sensitivity and specificity as pancreatic cancer biomarkers. ${ }^{285,286}$ To date, novel markers such as ncRNAs (miRNA and IncRNA), 287,288 genetic markers (e.g., KRAS, TP53, SMAD4, and CDKN2A), ${ }^{17,289}$ circulating tumor DNA (ctDNA), ${ }^{290}$ circulating tumor cells (CTCs), ${ }^{291,292}$ and exosomes ${ }^{293,294}$ have been explored. They show great potential for pancreatic cancer early detection, and might provide significant improvement in the future management and treatment outcome of pancreatic cancer. A liquid biopsy allows for serial, real-time monitoring of the dynamics and complex nature of cancer. In this section, we focus on the most promising liquid biopsy markers: CTCs, ctDNA, and exosomes, and their clinical applications in pancreatic cancer.

CTCs, which can be found in pancreatic cancer patients' circulation, provide useful information for diagnosis, staging, and prognosis, and they even can act as novel personalized treatment targets. $^{295,296}$ Some studies have reported that CTCs can be detected and used as an early-stage diagnostic marker using a number of methods, including negative enrichment, immunofluorescence and in situ hybridization (FISH) of chromosome (NEiFISH) system, and subtraction enrichment and immunostainingfluorescence in situ hybridization (SE-iFISH). NE-iFISH exhibits a very high CTC detection rate $(90 \%)$ in pancreatic cancer patients. SE-iFISH has both high sensitivity (88\%) and high specificity $(90 \%)$ in pancreatic cancer with a cutoff value of 2 cells/7.5 ml. ${ }^{297,298}$ CTCs in venous blood samples from pancreatic cancer patients were evaluated using a microfluidic NanoVelcro CTC chip and CTCs were detected in 54 out of 72 patients with a sensitivity and specificity of $75.0 \%$ and $96.4 \%$, respectively. ${ }^{299}$ Despite differences in CTCs enrichment and detection methods, studies have consistently shown that a higher number of CTCs could be predictive of reduced survival. ${ }^{297,300-302}$ In addition, a higher number of CTCs in the portal vein correlated well with a higher risk of cancer recurrence and liver metastases after surgery. For example, at a 3-year follow-up with patients who underwent surgical resection, portal vein CTC-positive patients presented a higher rate of liver metastases than CTC-negative patients. ${ }^{303}$ In another study, $84.6 \%$ of patients with a high portal CTCs count (defined as $>112 \mathrm{CTCs} / 2 \mathrm{ml}$ blood) developed liver metastases within 6 months after surgery. ${ }^{304}$ CTCs can also be a promising biomarker or useful for monitoring the response to chemotherapy of pancreatic cancer patients. It has been reported that triploid CTCs (which show three hybridization signals for a given chromosome) $<3$ groups displayed significantly increased 1-year survival and OS compared with the triploid CTCs $\geq 3$ group. ${ }^{298}$ Moreover, advanced pancreatic cancer patients can be tested for the presence of circulating tumor microemboli, whose presence predicts a low response to chemotherapy and poor survival. ${ }^{298,305}$
ctDNA, which represents a small fraction of the total cell-free DNAs (cfDNA), is derived from either the CTCs or the tumor cells. ${ }^{306}$ The sensitivity of ctDNA detection is generally lower than that of CA19-9 detection for early pancreatic cancer diagnosis. ${ }^{307}$ However, ctDNA proved to be a more suitable biomarker than CA19-9 and CTCs for the prognosis for pancreatic cancer patients. The higher burden of KRAS mutation on cfDNA levels has been shown to be negatively correlated with OS. ${ }^{308-310}$ Furthermore, the ctDNA level is a good indicator of pancreatic cancer progression during or after treatment (chemotherapy and/or surgery). Pancreatic cancer patients with detectable ctDNA showed poorer OS and a higher risk of cancer recurrence compared with those with undetectable ctDNA. ${ }^{37,311-313}$

Tumor-derived exosomes are enriched with DNA, mRNA, miRNA, and protein both inside and on their surface, establishing them as potential biomarkers for early-stage diagnosis of pancreatic cancer. ${ }^{314-316}$ Melo et al. reported that the biomarker glypican-1 (GPC1) was specifically enriched on pancreatic cancer exosomes. ${ }^{317} \mathrm{GPC} 1(+) \mathrm{GPC} 1$-circulating exosomes were detected in the serum of pancreatic cancer patients with $100 \%$ specificity and sensitivity, thereby distinguishing pancreatic cancer patients from patients with nonmalignant pancreatic disease and healthy subjects. ${ }^{317-319}$ In addition, certain exosomal miRNAs (miR-10b, $-21,-30 c,-181 a$, and -let7a) had $100 \%$ sensitivity and $100 \%$ specificity, and ephrin receptor A2-EV had $94 \%$ sensitivity and $85 \%$ specificity in distinguishing pancreatic cancer from healthy controls. ${ }^{320,321}$ With such high specificity and sensitivity, pancreatic tumor-derived exosomes might be one of the most promising markers for both early-stage pancreatic cancer diagnosis and monitoring of the therapeutic response during treatment. ${ }^{321-324}$

While liquid biopsies are increasingly being adopted for potential biomarkers for cancer diagnosis, prediction, and surveillance, there is a need for developments in assay technology for the isolation, quantification, and analysis of biomarkers based on liquid biopsy. For example, it is difficult to isolate tumorspecific DNA because of the limitations of the currently available techniques. ${ }^{325}$ Despite these shortcomings, significant research is still dedicated to liquid biopsies because of their potential advantages. One well-validated, Food and Drug Administration (FDA)-approved liquid biopsy test panel, MSK-IMPACT, was developed at the Memorial Sloan Kettering Cancer Center. They have also developed another liquid biopsy assay called the Analysis of Circulating Cell-free DNA to Evaluate Somatic Status. ${ }^{326}$ By using the latter panel, Razavi et al. identified cancer-derived somatic variants in plasma-circulating cfDNA and highlighted the importance of matched cfDNA white blood cell sequencing for accurate variant interpretation. ${ }^{327}$ This panel was also used to identify clinically relevant mutations and mutation signatures as well as novel noncoding alterations. ${ }^{328}$ This panel was subsequently expanded via Clinical Laboratory Improvement Amendment-certified testing for the analysis of tumor-derived and matched germline DNA samples, which has also been approved by the FDA. ${ }^{329}$ However, the majority of liquid biopsy assays still lack sufficient evidence of clinical validity and utility. Only after the demonstration of clinical validity and clinical utility, liquid biopsies may further reach their full potential and impact on the clinical management of pancreatic cancer patients.

Subtype-specific therapeutic responses in pancreatic cancer Pancreatic cancer is extensive heterogeneity amongst patients with respect to treatment response. Basal-like tumors have a higher frequency of TP53 mutations, ${ }^{36,46,48}$ while the classical subtype is characterized by GATA binding protein 6 (GATA6) expression and KRAS dependency. ${ }^{44,46}$ Importantly, patients with basal-like tumors have higher pathologic grades and worse OS compared with those with classical tumors. ${ }^{44,46,48,50,330}$ Nevertheless, there is no consensus on subtype-specific treatment regimens. Although Collisson et al. reported that QM-PDA subtype 
patients had significantly worse outcomes compared with patients with other subtypes, they found that in pancreatic cancer cells, the QM-PDA subtype was more sensitive to gemcitabine. By contrast, classical subtype cell lines were more sensitive to erlotinib. ${ }^{44}$ One possible explanation for these findings is that in vitro 2D-cultured cell lines might not manifest expected bulk RNA-sequencing (RNAseq(-based molecular signatures, as Moffitt et al. reported that traditional 2D-cultured cell lines lack the classical subtype. ${ }^{47}$ Moffitt et al. also demonstrated that patients with basal-like tumors showed a tendency to benefit more from adjuvant therapy, although the effect was not significant. ${ }^{47}$ In vivo patient-derived xenograft (PDX) models or 3D organoid-based approaches might be more effective tools for investigating subtype-specific treatment responses. ${ }^{45}$ Interestingly, by using an organoid-based model, Tiriac et al. found that the basal-like subtype was enriched in an oxaliplatin-nonsensitive group, while it was present at similar frequencies in gemcitabine-sensitive and nonsensitive groups. ${ }^{45}$

Recently, Aung et al. reported the results of the COMPASS trial, showing that basal-like subtype tumors showed a poorer response to first-line chemotherapy (1/12 vs. $13 / 38$ had a partial response in the basal-like vs. classical subtype, respectively). Patients with classical subtype tumors treated with mFOLFIRINOX had the best PFS. ${ }^{331}$ More recently, Chan et al. devised a more comprehensive method for molecular subtyping and for developing subtypespecific treatment regimens in pancreatic cancer. ${ }^{46}$ They reclassified pancreatic cancer samples into five subtypes: basal-like $A$, basal-like $B$, hybrid, classical $A$, and classical $B$. Distinguishing the basal-like $A$, basal-like $B$, and hybrid subtypes from the former basal-like/squamous subtype allowed the detection of more subtle subtype-specific chemotherapy responses; furthermore, Chan et al. reported that the basal-like $A$ subtype showed the poorest responses to gemcitabine-based and mFOLFIRINOX chemotherapies. ${ }^{46}$ Remarkably, single-cell RNA-seq data provided evidence of the coexistence of basal-like and classical expression signatures within a single cancer sample; furthermore, subtype switching, a rare event due to the outgrowth of minor clones driven by therapeutic selection and genetic instability, was observed. ${ }^{46}$ Additional evidence of intratumoral transcriptional heterogeneity in pancreatic cancer was found by Hayashi et al. via multiregional sampling. ${ }^{332}$ In addition, $\mathrm{Er}$ et al. demonstrated a squamous subtype-specific synergistic effect of Src and MEK1/2 inhibitors with gemcitabine in pancreatic cancer cell lines. ${ }^{333}$

Limited access to freshly frozen samples and cost-prohibitive issues have limited the use of transcriptomic-based subtyping. Puleo et al. evaluated the transcriptome of formalin-fixed paraffinembedded pancreatic cancer samples and successfully identified basal-like and classical subtypes. ${ }^{48}$ Law et al. reported proteomicbased subtyping of pancreatic cancer liver metastases and identified four distinct pancreatic cancer subtypes, i.e., metabolic, progenitor-like, proliferative, and inflammatory. ${ }^{30}$ Pancreatic cancer patients with metabolic and progenitor-like subtypes showed significant benefit from FOLFIRINOX treatment.

Hayashi et al. and Kalimuthu et al. provided paradigms for integrating clinical morphological and histological features with transcriptomic expression profiles such that pancreatic cancer molecular subtypes can be predicted based on morphological classification. ${ }^{332,334}$ Hayashi et al. found that histological squamous features and glandular patterns were consistent with the RNA-seq-defined basal-like and classical subtypes, respectively. ${ }^{332}$ They also identified intratumoral heterogeneity in transcriptional subtypes and suggested a subclonal feature of pancreatic cancer subtypes, which was inconsistent with Chan et al. ${ }^{46}$ Kalimuthu et al. identified four morphological patterns that segregated into two components, i.e., gland-forming and non-gland-forming, that corresponded to the classical and basal-like subtypes. ${ }^{334}$ They even found that morphological pattern-based subgroups provide better prediction of clinical outcomes than transcriptional subtypes. Efforts have also been made to simplify the classification of pancreatic cancer samples using surrogate markers, e.g., TP63, ${ }^{335}$ YAP1, ${ }^{92}$ HNF1 homeobox $A$, keratin 81 (KRT81), GATA6, ${ }^{44,46}$ and $K R T 17 .{ }^{336}$ Interestingly, Kaissis et al. reported that a machine learning-based analysis of preoperative CT images could predict molecular pancreatic cancer subtypes. ${ }^{337}$ These findings might facilitate clinical patient stratification and provide guidance for precision medicine.

Although some studies have demonstrated the feasibility of prospective genomic profiling of advanced pancreatic cancer, this approach is time-consuming and costly. ${ }^{331,338}$ In addition, clustering algorithms are threshold sensitive, and molecular classifiers are heavily confounded by limitations such as sampling methods, tumor cellularity, RNA quality, and tumor heterogeneity. ${ }^{338,339}$ Nevertheless, molecular subtyping now guides the optimization of therapeutic strategies and provides valuable opportunities for improving pancreatic cancer patient outcomes.

Current preclinical and clinical developments in pancreatic cancer Current preclinical therapeutic models in pancreatic cancer. Identification of clinically applicable approaches for pancreatic cancer treatment relies heavily on the availability of preclinical models. In recent decades, novel preclinical models, e.g., PDX systems and patient-derived organoids (PDO), have been developed and are becoming increasingly used in drug screening, biomarker development, and evaluation of personalized therapeutic strategies. PDX and PDO models have positive predictive values of 80 and $88 \%$ for patient-specific sensitivities to therapeutic agents, respectively. ${ }^{340-342}$ However, PDX and PDO models are primarily suitable for basic research and have severe limitations for clinical use. For example, it normally takes several months for a PDX model to yield preclinical results. Moreover, it is infeasible to study early carcinogenesis, progression, and tumor immunology based on PDX or PDO models.

Recently, advances in genetic engineering techniques and an increased understanding of the mechanisms of pancreatic oncogenesis have supported the development of GEMMs useful as pancreatic cancer preclinical models (Table 1). Most of the GEMMs currently used in translational oncological studies are Cre/ loxP-based models. Endogenous expression of oncogenic $K R A S^{G 12 D}$ induces PanIN in mice, and a subset of these mice develop pancreatic cancer tumors at an advanced age, revealing that additional events are necessary for tumor formation onset. ${ }^{343}$

Strains for targeting pancreatic progenitor cells, such as Pdx1Cre transgenic or Ptfla $a^{+/ C r e}$ knock-in strains in the context of endogenous mutant KRAS ${ }^{G 12 D}$ (generally referred to as KC mice) have been successfully used to recapitulate the development and progression of PanIN lesions to pancreatic cancer. ${ }^{34,344}$ Furthermore, the KPC PDAC mouse model, first described in 2005, allows conditional activation of the KRAS ${ }^{G 12 D}$ mutation and a TP53 mutation (Pdx1-Cre; LSL-KRAS ${ }^{G 12 D /+}$; LSL-TP53 ${ }^{R 172 H /+}$ ) via Cre-Lox technology. ${ }^{345}$ In addition, combined with the KRAS ${ }^{G 12 D}$ mutation, Ink4a/Arf deficiency (Pdx1-Cre; LSL-KRAS ${ }^{G 12 D /+}$; Ink4a/Arf $\left.{ }^{\text {lox/lox }}\right)$, ATM deficiency (Ptf1 $\left.a^{+/ C r e} ; K_{R A S}{ }^{G 12 D /+} ; A T M^{\text {lox/lox }}\right)$, or a TP53 $3^{\text {lox/lox }}$ background plus $P^{\prime} 16^{\operatorname{lnk} 4 a}$ deficiency (Pdx1-Cre; KRAS ${ }^{G 12 D /+}$; Ink $4 a^{-1-}$ TP53 ${ }^{\text {lox/lox }}$ ) result in accelerated progression from PanIN to invasive PDAC. ${ }^{345-349}$ Concomitant expression of TGFa and $K R A S^{G 12 D}$ leads to the development of cystic papillary lesions that resemble human IPMN (KRAS ${ }^{G 12 D /+}$; Ela-TGFa). ${ }^{350}$ Concomitant presence of KRAS G12D and SMAD deficiency (Pdx1-Cre; KRAS ${ }^{G 12 D /+}$; Smad lox/lox or Ptf1a ${ }^{+/ C r e}$; KRAS ${ }^{G 12 D /+}$; Smad ${ }^{\text {lox/lox }}$ ) leads to the development of IPMN or mucinous cystic neoplasm, respectively. ${ }^{351,352}$ Compared with traditional xenograft models, these GEMMs more faithfully recapitulate key morphological and molecular PDAC features. They can be used to study early carcinogenesis, progression, and tumor immunology of traditional xenograft models. GEMM models also offer higher predictive value for clinical diagnostic and therapeutic interventions. However, 
despite the increasing repertoire of animal models that emulate key genetic driver mutations found in pancreatic cancer, it is important to emphasize that many GEMMs do not fully recapitulate all aspects of the clinical population by fully covering the full range of genetic diversity and metastatic spread, as demonstrated by multiple failed clinical trials. ${ }^{353,354}$

Clinical strategies in pancreatic cancer. For pancreatic cancer patients, the only potentially curative choice is surgical resection of the pancreas. This approach is restricted to $20 \%$ of cases with anatomically resectable disease. Moreover, up to $50 \%$ of patients have incomplete resection accompanied by positive surgical margins. For those patients, the overall 5 -year survival sharply decreases to $7 \% .^{16,355,356}$ It has been demonstrated that adjuvant chemotherapy can improve OS, but postoperative complications limit the intended treatment in $50 \%$ of patients. ${ }^{357}$ For patients with borderline or locally advanced pancreatic cancer, neoadjuvant therapy can convert unresectable disease into a potentially resectable state, which benefits OS. $^{358}$

Current guidelines recommend FOLFIRINOX, mFOLFIRINOX, gemcitabine, or gemcitabine plus nab-paclitaxel for preoperative pancreatic cancer treatment. From 1997 to 2011, the first-line chemotherapy for patients with advanced pancreatic cancer was gemcitabine monotherapy. ${ }^{10}$ The treatment method changed in 2011 when FOLFIRINOX demonstrated a better survival benefit compared with that of gemcitabine monotherapy. ${ }^{11}$ Thereafter, gemcitabine plus nab-paclitaxel was adopted as another first-line treatment option. This treatment method had a better survival benefit (median OS 8.5 vs. 6.7 months, hazard ratio $0.72,95 \%$ confidence interval $0.62-0.83, P<0.001$ ) compared with gemcitabine monotherapy. ${ }^{12}$ According to a large-scale retrospective study, FOLFIRINOX and nab-paclitaxel plus gemcitabine have similar outcomes. ${ }^{359}$ However, these therapeutic methods are reserved for otherwise healthy patients with good performance status. For the elderly and patients with a poor performance status, gemcitabine monotherapy is still considered a more tolerable treatment. ${ }^{360}$ The updated pancreatic cancer treatment guidelines recommend pembrolizumab immunotherapy for patients that carry mismatch repair (MMR) deficiencies or microsatellite instability after the failure of first-line therapies. ${ }^{361}$ As a second-line treatment option for pancreatic cancer, a combination of 5-FU and liposomal irinotecan is the only approved therapeutic method. ${ }^{362}$ In addition to these cytotoxic chemotherapies, novel agents are also under active investigation. The addition of leucovorin to S-1 improved PFS in patients with gemcitabine-refractory advanced pancreatic cancer. $^{363}$ For patients with pancreatic cancer, novel therapeutic trials targeting DNA repair, gene mutations, tumor metabolism, the tumor microenvironment, or immune checkpoints might improve their prognosis (Fig. 4 and Table 2).

The high prevalence of KRAS mutations (carried by $>90 \%$ of pancreatic cancer patients) has led to considerable interest in KRAS-targeted therapies. Unfortunately, current approaches for direct targeting of mutant KRAS protein are ineffective due to their high affinity for GTP and/or GDP. ${ }^{36,364}$ An alternative strategy was developed to target KRAS by using exosomes or small EVs loaded with small interfering RNAs that target KRAS ${ }^{G 12 D}$. This study has entered a phase 1 clinical trial for patients with metastatic pancreatic cancer (NCT03608631). ${ }^{365}$ Furthermore, the National Cancer Institute in the United States also established the RAS Initiative in 2013 to explore effective therapies for RAS-related cancers (https://www.cancer.gov/research/key-initiatives/ras).

Increasing interest has also emerged in the targeting of lowprevalence, actionable aberrations, including BRCA1/2, NTRK1/2/3, or MMR deficiencies. In three phase 1 and 2 clinical trials evaluating the treatment of patients with advanced or metastatic NTRK fusion-positive solid tumors with a potent TRK inhibitor, entrectinib, a complete response (CR) of $7 \%$ and a partial response 


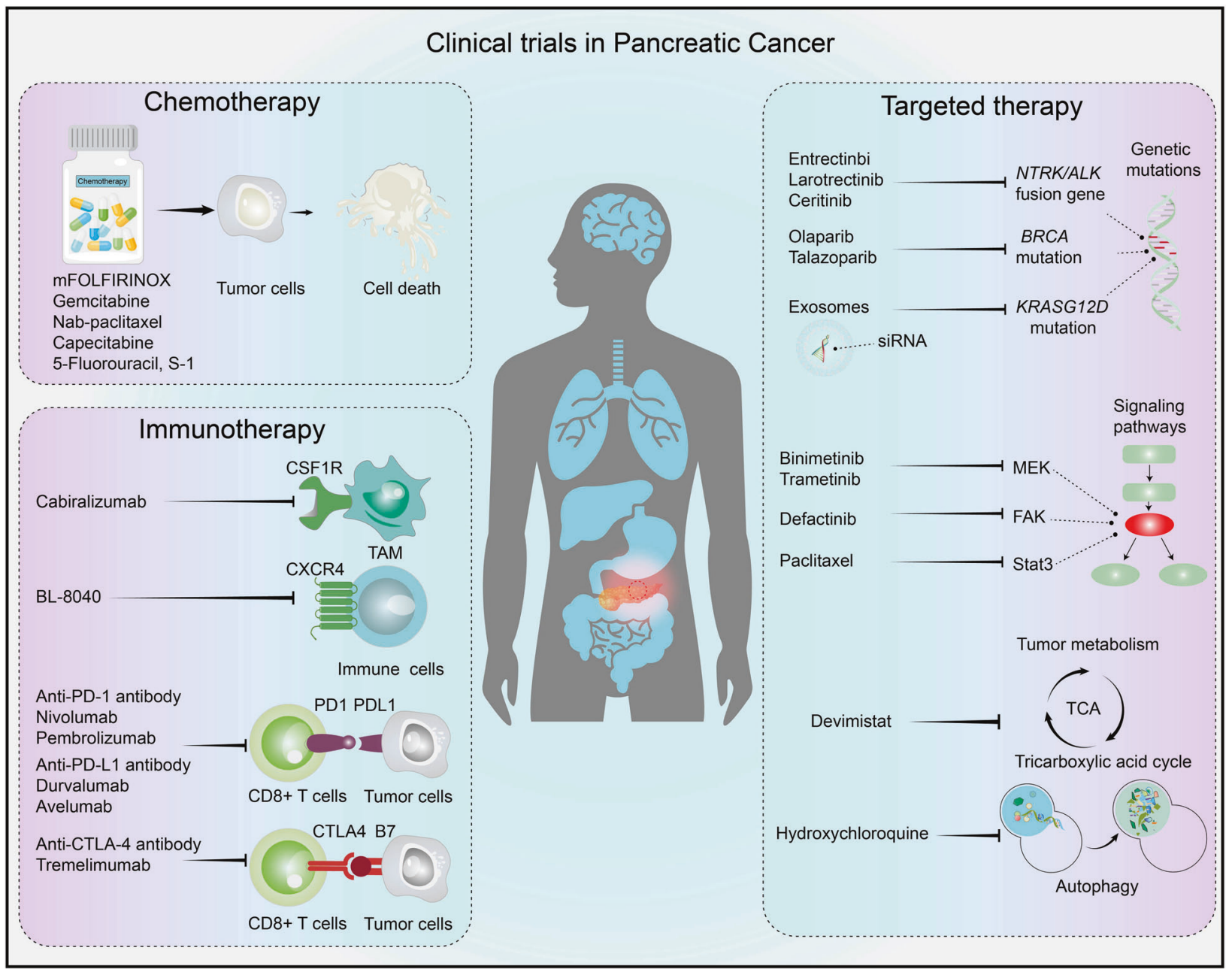

Fig. 4 Current clinical strategies in pancreatic cancer. For patients with pancreatic cancer, the primary clinical strategies rely on chemotherapy, whereas novel therapeutic agents targeting DNA repair, gene mutations, tumor metabolism, tumor microenvironments, or immune checkpoints might improve their prognosis. Currently, increasing interest has emerged in combined chemotherapy and immunotherapy or targeted therapy

(PR) of 50\% were achieved (NCT02097810, NCT02568267, EudraCT 2012-000148-88). ${ }^{366}$ In other clinical trials evaluating the efficacy of the TRK inhibitor larotrecitinib, the objective response rate (ORR) reached $75 \%$ among 55 patients with NTRK fusion-positive tumors, including one pancreatic cancer patient who had a PR (NCT02122913, NCT02637687, NCT02576431). ${ }^{367}$ Larotrectinib and entrectinib received FDA approval for use as tissue-agnostic indicators in patients with solid tumors with NTRK fusions in November 2018 and August 2019, respectively. Furthermore, the use of ALK inhibitors in pancreatic cancer patients is currently under investigation. ALK rearrangements have also been established as a promising molecular target in other malignancies, e.g., non-small cell lung cancer. ${ }^{368}$ In a 2017 study, five cases harboring an ALK fusion gene were identified via comprehensive genomic profiling of 3170 pancreatic cancer patients. Among them, four patients were treated with an ALK inhibitor, three of which showed a stable radiographic response and/or normalization of serum CA19-9. ${ }^{369}$ A phase 1 study to combine ceritinib, a novel ALK inhibitor, with chemotherapy for the treatment of advanced pancreatic cancer has just completed (NCT02227940).

Therapeutic strategies based on BRCA-poly (ADP-ribose) polymerase (PARP) synthetic lethality have shown to be effective for the treatment of patients with BRCA1/2 mutations. A multicenter phase 2 trial of olaparib (a PARP inhibitor) for the treatment of patients with a germline BRCA1/2 mutation and recurrent cancer achieved an ORR of $26.2 \%$ overall and an ORR of $21.7 \%$ for pancreatic cancer (NCT01078662). ${ }^{370}$ In the POLO trial and a randomized phase 3 trial for patients with metastatic pancreatic cancer with a germline BRCA1/2 mutation after at least 16 weeks of platinum-based chemotherapy, olaparib as a maintenance therapy achieved an ORR of $20 \%$ with a PFS median of 7.4 months, compared with $10 \%$ and 3.8 months in the placebo group (NCT02184195). ${ }^{14}$

The tumor microenvironment is another area of interest in pancreatic cancer therapeutic exploration. Immune checkpoint inhibitors (ICls) have emerged as a new treatment paradigm for patients with certain types of tumors. However, the results of early clinical trials investigating the efficacy of the anti-CTLA-4 antibody ipilimumab or the anti-PD-L1 antibody BMS-936559 in patients with advanced pancreatic cancer were disappointing. ${ }^{371,372}$ In the phase 2 KEYNOTE-158 study of pembrolizumab (an anti-PD-1 monoclonal antibody), an ORR of $34.3 \%$ was achieved in patients with previously treated, advanced non-colorectal high microsatellite instability (MSI-H)/DNA MMR (dMMR)-deficient cancer, and in pancreatic cancer patients, an ORR of $18.2 \%$, a CR of $4.5 \%$, and a PR of $13.6 \%$ were achieved (NCT02628067). ${ }^{373}$ Based on these results, the FDA granted accelerated approval to pembrolizumab for adult and pediatric patients with unresectable MSI-H or dMMR solid tumors in May 2017. Clinical trials of PD-1 blockade or PD-L1 combined with other $\mathrm{ICls}$ or blockade are currently ongoing 


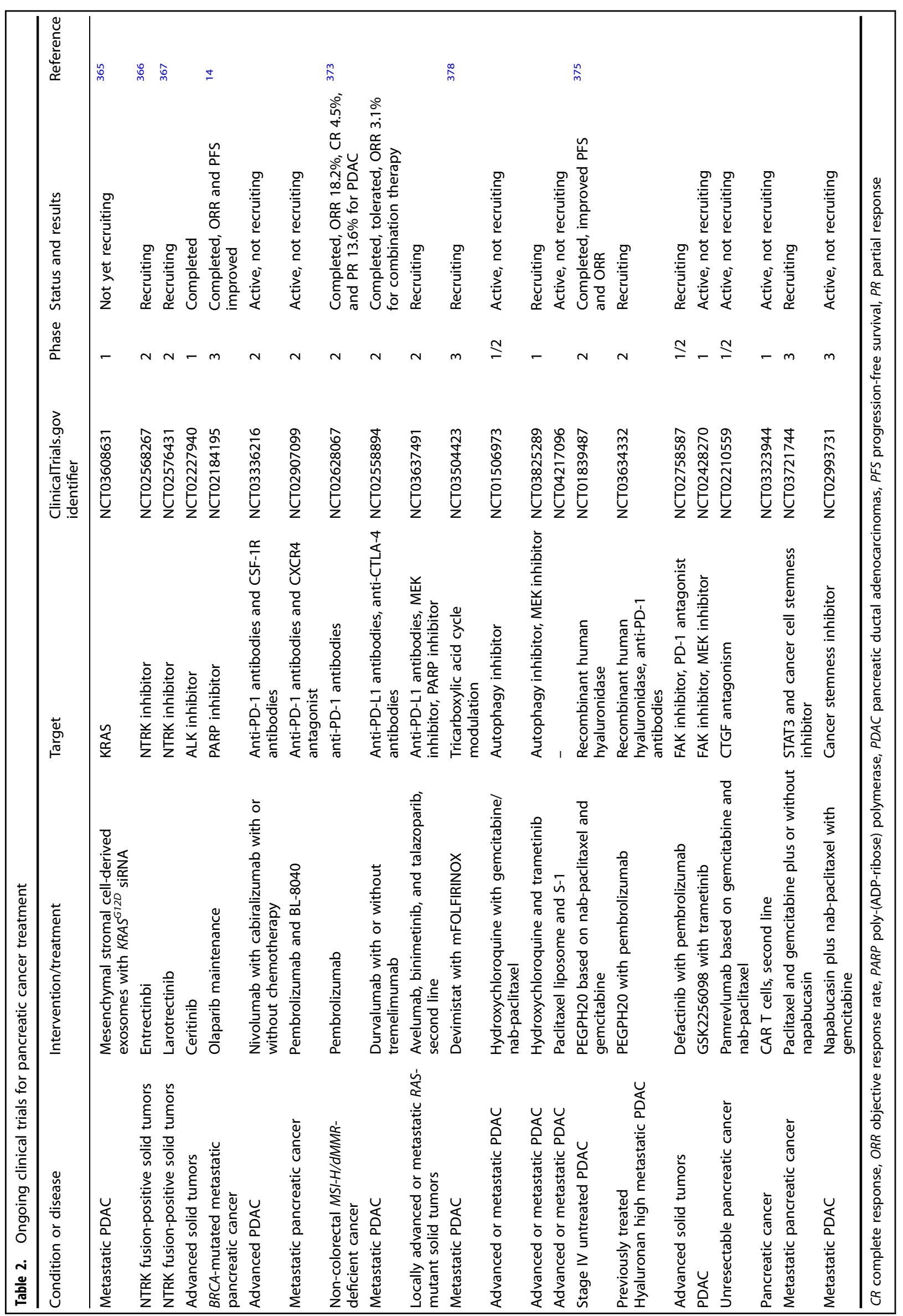


(NCT03336216, NCT02907099, NCT02558894, NCT03637491). Hyaluronic acid is a hydrophilic glycosaminoglycan whose overproduction in the stroma of patients with pancreatic cancer leads to increased interstitial tumor pressure, reducing tumor perfusion and access of anticancer drugs to the tumor. ${ }^{374} \mathrm{~A}$ phase 2 trial showed that the addition of PEGPH2O (pegvorhyaluronidase-a, recombinant human hyaluronidase) to nab-paclitaxel and gemcitabine resulted in significant improvements in ORR (45 vs. $31 \%$ ) and PFS (9.2 vs. 5.2 months) in pancreatic cancer patients with high hyaluronic acid expression levels (NCT01839487). ${ }^{375}$ However, a later phase 3 study failed to show improvements in OS or PFS, and the results did not support additional development of PEGPH20 as a treatment for metastatic PDAC, as a high rate of dose holds and reductions in the PEGPH20 arm might have led to lower chemotherapy drug exposure, which could have contributed to the inferior survival outcomes with PEGPH20. ${ }^{376}$

Metabolic reprogramming can also be a therapeutic target in pancreatic cancer. Devimistat, which inhibits enzymes in the mitochondrial tricarboxylic acid cycle, is hypothesized to act synergistically with cytotoxic agents to induce decreased production of anabolic intermediates required for DNA damage repair. A phase 1 study of devimistat combined with mFOFIRINOX for the treatment of metastatic pancreatic cancer patients showed an ORR of $61 \%$, including a CRR of $17 \%$ (NCT01835041). ${ }^{377}$ Additional clinical trials evaluating the safety and efficacy of devimistat combined with gemcitabine and nab-paclitaxel or mFOLFIRINOX are ongoing (NCT03435289, NCT03504423). ${ }^{378}$ Inhibition of autophagy in pancreatic cancer primary tumors contributes to a metabolic defect that leads to decreased mitochondrial OXPHOS and significant growth suppression. ${ }^{213}$ In a phase 2 study, autophagy inhibition using HCQ in patients with metastatic pancreatic cancer failed to achieve therapeutic efficacy. ${ }^{379}$ Further clinical trials to test the effectiveness of HCQ combined with cytotoxic agents or MEK inhibitors are undergoing (NCT01506973, NCT03825289).

A number of combined therapeutic approaches have already been introduced. Preclinical research has shown that FAK inhibition can lead to decreased levels of fibrosis, synergy with chemotherapy and ICls, and improved survival outcomes in the KPC mouse model of pancreatic cancer. ${ }^{254}$ Currently, a number of clinical trials to investigate the efficacy of combinations of FAK inhibitors and ICls and/or cytotoxic agents or MEK inhibitors are ongoing (NCT02546531, NCT02758587, NCT02428270). Connective tissue growth factor (CTGF), a member of the CCN family of secreted proteins, is involved in ECM production, desmoplasia, and tumor progression. ${ }^{380}$ Preclinical data indicated that CTGF antagonism with the therapeutic monoclonal human antibody pamrevlumab enhanced the response to gemcitabine in pancreatic cancer mouse models. ${ }^{381}$ The safety and efficacy of the agent in combination with gemcitabine and nab-paclitaxel for the treatment of patients with unresectable pancreatic cancer were evaluated in a phase $1 / 2$ trial (NCT02210559). The results indicated that the addition of pamrevlumab to neoadjuvant therapy in locally advanced pancreatic cancer patients might lead to higher resectability and resection rate. Currently, two phase 3 studies to investigate the efficacy of the addition of napabucasin to gemcitabine and nab-paclitaxel for treating metastatic pancreatic cancer patients are ongoing (NCT03721744, NCT02993731). ${ }^{382}$

Preclinical investigations in pancreatic cancer PDX models identified a small subset of tumor cells (termed CSCs) that determines self-renewal and metastatic phenotypes of pancreatic cancer. ${ }^{383}$ A phase $1 \mathrm{~b} / 2$ study of the cancer stemness inhibitor napabucasin combined with gemcitabine and nab-paclitaxel for treating metastatic pancreatic cancer achieved a CR of $3 \%$ and a PR of $42 \%$.

In summary, for patients with resectable pancreatic cancer, mFOLFIRINOX and nab-paclitaxel plus gemcitabine regimens were shown to achieve the longest median OS. For patients with advanced or metastatic pancreatic cancer, treatment regimens based on the identification of potentially actionable alterations in small subsets of patients via comprehensive genomic profiling and monitoring of the efficacy of therapeutic strategies are currently being explored. The POLO trial, which involved the identification of germline BRCA mutations and targeted therapy, proved to be effective, and such approaches are worth further exploration.

\section{CONCLUSIONS AND FUTURE PERSPECTIVES}

Pancreatic cancer remains one of the most common and deadly cancers with limited options for effective therapy. Meaningful clinical progress in diagnostic investigations, surgical techniques, and systemic therapies are certain to improve pancreatic cancer patient survival. A deeper understanding of the biology and genetics of pancreatic cancer, including new insight into driver gene mutations, tumor metabolism, and the tumor microenvironment, might lead to promising and innovative therapeutic strategies. It has been widely proposed that targeting a single molecule or pathway is unlikely to yield more pancreatic cancer therapies. Both subtype-specific therapy and combined therapy might represent more promising strategies to control tumor progression. Recent advances showing that pancreatic cancer patients with germline BRCA mutations benefit from PARP inhibitors might inspire novel strategies that further increase the clinical efficacy of subtype-specific therapy. While clinical progress has never been clearer in the improvement of outcomes in pancreatic cancer patients, the path to clinical translation of novel therapeutic approaches will be greatly enhanced by the use of more sophisticated animal models and multidisciplinary clinical collaborations.

\section{ACKNOWLEDGEMENTS}

This work was supported by the following: the National Key Research and Development Program of China (No. 2017YFC1308604); National Natural Science Foundation of China (Nos. 81802903, 81672820, 81940074, and 81872356); the Program of Shanghai Academic Research Leader (No. 20XD1400900); the Shanghai International Science and Technology Collaboration Program.

\section{AUTHOR CONTRIBUTIONS}

Q.Z.-D., L.-X.Q., and Y.Z. designed the review. S.W., Y.Z., and F.Y. drafted the manuscript. L.Z., X.-Q.Z., Z.-F.W., X.-L.W., C.-H.Z., J.-Y.Y., B.-Y.H., B.K., and D.-L.F. prepared the figures. C.B., L.-X.Q., and Q.-Z.D. participated in its coordination and modification. All authors read and approved the final manuscript.

\section{ADDITIONAL INFORMATION}

Competing interests: The authors declare no competing interests.

\section{REFERENCES}

1. Sung, H. et al. Global Cancer Statistics 2020: GLOBOCAN Estimates of Incidence and Mortality Worldwide for 36 Cancers in 185 Countries. CA Cancer J. Clin. 71, 209-249 (2021).

2. Siegel, R. L., Miller, K. D., Fuchs, H. E. \& Jemal, A. Cancer statistics, 2021. CA Cancer J. Clin. 71, 7-33 (2021).

3. Miller, K. D. et al. Cancer statistics for adolescents and young adults, 2020. CA Cancer J. Clin. 70, 443-459 (2020).

4. Rawla, P., Sunkara, T. \& Gaduputi, V. Epidemiology of pancreatic cancer: global trends, etiology and risk factors. World J. Oncol. 10, 10-27 (2019).

5. Abe, T. et al. Deleterious germline mutations are a risk factor for neoplastic progression among high-risk individuals undergoing pancreatic surveillance. $J$. Clin. Oncol. 37, 1070-1080 (2019).

6. Kromrey, M. L. et al. Prospective study on the incidence, prevalence and 5-year pancreatic-related mortality of pancreatic cysts in a population-based study. Gut 67, 138-145 (2018)

7. Kleeff, J. et al. Pancreatic cancer. Nat. Rev. Dis. Prim. 2, 16022 (2016). 
8. Kuehn, B. M. Looking to long-term survivors for improved pancreatic cancer treatment. JAMA 324, 2242-2244 (2020).

9. Picozzi, V. J. et al. Five-year actual overall survival in resected pancreatic cancer: a contemporary single-institution experience from a multidisciplinary perspective. Ann. Surg. Oncol. 24, 1722-1730 (2017).

10. Burris, H. A. 3rd et al. Improvements in survival and clinical benefit with gemcitabine as first-line therapy for patients with advanced pancreas cancer: a randomized trial. J. Clin. Oncol. 15, 2403-2413 (1997).

11. Conroy, T. et al. FOLFIRINOX versus gemcitabine for metastatic pancreatic cancer. N. Engl. J. Med. 364, 1817-1825 (2011).

12. Von Hoff, D. D. et al. Increased survival in pancreatic cancer with nab-paclitaxel plus gemcitabine. N. Engl. J. Med. 369, 1691-1703 (2013).

13. Conroy, T. et al. FOLFIRINOX or gemcitabine as adjuvant therapy for pancreatic cancer. N. Engl. J. Med. 379, 2395-2406 (2018).

14. Golan, T. et al. Maintenance olaparib for germline BRCA-mutated metastatic pancreatic cancer. N. Engl. J. Med. 381, 317-327 (2019).

15. Assenat, E. et al. Phase II study evaluating the association of gemcitabine, trastuzumab and erlotinib as first-line treatment in patients with metastatic pancreatic adenocarcinoma (GATE 1). Int. J. Cancer 148, 682-691 (2021).

16. Mizrahi, J. D., Surana, R., Valle, J. W. \& Shroff, R. T. Pancreatic cancer. Lancet 395 2008-2020 (2020).

17. Buscail, L., Bournet, B. \& Cordelier, P. Role of oncogenic KRAS in the diagnosis, prognosis and treatment of pancreatic cancer. Nat. Rev. Gastroenterol. Hepatol. 17, 153-168 (2020).

18. Singh, K. et al. Kras mutation rate precisely orchestrates ductal derived pancreatic intraepithelial neoplasia and pancreatic cancer. Lab. Invest. 101, 177-192 (2021).

19. Prior, I. A., Lewis, P. D. \& Mattos, C. A comprehensive survey of Ras mutations in cancer. Cancer Res. 72, 2457-2467 (2012).

20. Witkiewicz, A. K. et al. Whole-exome sequencing of pancreatic cancer defines genetic diversity and therapeutic targets. Nat. Commun. 6, 6744 (2015).

21. Mann, K. M., Ying, H., Juan, J., Jenkins, N. A. \& Copeland, N. G. KRAS-related proteins in pancreatic cancer. Pharm. Ther. 168, 29-42 (2016).

22. Qian, Y. et al. Molecular alterations and targeted therapy in pancreatic ducta adenocarcinoma. J. Hematol. Oncol. 13, 130 (2020).

23. Christenson, E. S., Jaffee, E. \& Azad, N. S. Current and emerging therapies for patients with advanced pancreatic ductal adenocarcinoma: a bright future. Lancet Oncol. 21, e135-e145 (2020).

24. Connor, A. A. et al. Integration of genomic and transcriptional features in pancreatic cancer reveals increased cell cycle progression in metastases. Cancer Cell 35, 267-282.e267 (2019).

25. Waddell, N. et al. Whole genomes redefine the mutational landscape of pancreatic cancer. Nature 518, 495-501 (2015).

26. Dardare, J., Witz, A., Merlin, J. L., Gilson, P. \& Harlé, A. SMAD4 and the TGF $\beta$ pathway in patients with pancreatic ductal adenocarcinoma. Int. J. Mol. Sci. 21, 3534 (2020)

27. Yachida, S. et al. Clinical significance of the genetic landscape of pancreatic cancer and implications for identification of potential long-term survivors. Clin. Cancer Res. 18, 6339-6347 (2012).

28. Ying, $\mathrm{H}$. et al. Genetics and biology of pancreatic ductal adenocarcinoma. Genes Dev. 30, 355-385 (2016).

29. Shen, G. Q., Aleassa, E. M., Walsh, R. M. \& Morris-Stiff, G. Next-generation sequencing in pancreatic cancer. Pancreas 48, 739-748 (2019).

30. Holter, S. et al. Germline BRCA mutations in a large clinic-based cohort of patients with pancreatic adenocarcinoma. J. Clin. Oncol. 33, 3124-3129 (2015)

31. Murphy, K. M. et al. Evaluation of candidate genes MAP2K4, MADH4, ACVR1B and BRCA2 in familial pancreatic cancer: deleterious BRCA2 mutations in $17 \%$. Cancer Res. 62, 3789-3793 (2002).

32. Tanaka, M. et al. Revisions of international consensus Fukuoka guidelines for the management of IPMN of the pancreas. Pancreatology 17, 738-753 (2017).

33. Chandana, S., Babiker, H. M. \& Mahadevan, D. Therapeutic trends in pancreatic ductal adenocarcinoma (PDAC). Expert Opin. Investig. Drugs 28, 161-177 (2019).

34. Feigin, M. E. et al. Recurrent noncoding regulatory mutations in pancreatic ductal adenocarcinoma. Nat. Genet. 49, 825-833 (2017).

35. Hung, Y. H., Hsu, M. C., Chen, L. T., Hung, W. C. \& Pan, M. R. Alteration of epigenetic modifiers in pancreatic cancer and its clinical implication. J. Clin. Med. 8, 903 (2019).

36. Bailey, P. et al. Genomic analyses identify molecular subtypes of pancreatic cancer. Nature 531, 47-52 (2016).

37. Sausen, M. et al. Clinical implications of genomic alterations in the tumour and circulation of pancreatic cancer patients. Nat. Commun. 6, 7686 (2015).

38. Lee, M. G. et al. Demethylation of $\mathrm{H} 3 \mathrm{~K} 27$ regulates polycomb recruitment and H2A ubiquitination. Science 318, 447-450 (2007).
39. Wang, M. \& Lin, H. Understanding the function of mammalian sirtuins and protein lysine acylation. Annu. Rev. Biochem. https://doi.org/10.1146/annurevbiochem-082520-125411 (2021).

40. Fritsche, P. et al. HDAC2 mediates therapeutic resistance of pancreatic cancer cells via the BH3-only protein NOXA. Gut 58, 1399-1409 (2009).

41. Aghdassi, A. et al. Recruitment of histone deacetylases HDAC1 and HDAC2 by the transcriptional repressor ZEB1 downregulates E-cadherin expression in pancreatic cancer. Gut 61, 439-448 (2012).

42. Mishra, V. K. et al. Histone deacetylase class-I inhibition promotes epithelial gene expression in pancreatic cancer cells in a BRD4- and MYC-dependent manner. Nucleic Acids Res. 45, 6334-6349 (2017).

43. Kugel, S. et al. SIRT6 suppresses pancreatic cancer through control of Lin28b. Cell 165, 1401-1415 (2016).

44. Collisson, E. A. et al. Subtypes of pancreatic ductal adenocarcinoma and their differing responses to therapy. Nat. Med. 17, 500-503 (2011).

45. Tiriac, $H$. et al. Organoid profiling identifies common responders to chemotherapy in pancreatic cancer. Cancer Discov. 8, 1112-1129 (2018).

46. Chan-Seng-Yue, M. et al. Transcription phenotypes of pancreatic cancer are driven by genomic events during tumor evolution. Nat. Genet. 52, 231-240 (2020).

47. Moffitt, R. A. et al. Virtual microdissection identifies distinct tumor- and stromaspecific subtypes of pancreatic ductal adenocarcinoma. Nat. Genet. 47 1168-1178 (2015)

48. Puleo, F. et al. Stratification of pancreatic ductal adenocarcinomas based on tumor and microenvironment features. Gastroenterology 155, 1999-2013.e1993 (2018).

49. Cancer Genome Atlas Research Network. Integrated genomic characterization of pancreatic ductal adenocarcinoma. Cancer Cell 32, 185-203.e113 (2017).

50. Noll, E. M. et al. CYP3A5 mediates basal and acquired therapy resistance in different subtypes of pancreatic ductal adenocarcinoma. Nat. Med. 22, 278-287 (2016).

51. Knudsen, E. S. et al. Pancreatic cancer cell lines as patient-derived avatars: genetic characterisation and functional utility. Gut 67, 508-520 (2018).

52. Maurer, $C$. et al. Experimental microdissection enables functional harmonisation of pancreatic cancer subtypes. Gut 68, 1034-1043 (2019).

53. Pylayeva-Gupta, Y., Grabocka, E. \& Bar-Sagi, D. RAS oncogenes: weaving a tumorigenic web. Nat. Rev. Cancer 11, 761-774 (2011).

54. Tago, K. et al. Oncogenic Ras mutant causes the hyperactivation of NF-KB via acceleration of its transcriptional activation. Mol. Oncol. 13, 2493-2510 (2019).

55. Loncle, C. et al. IL17 functions through the novel REG3 $\beta$-JAK2-STAT3 inflammatory pathway to promote the transition from chronic pancreatitis to pancreatic cancer. Cancer Res. 75, 4852-4862 (2015).

56. Baumgart, S. et al. GSK-3 $\beta$ Governs Inflammation-Induced NFATc2 Signaling Hubs to Promote Pancreatic Cancer Progression. Mol. Cancer Ther. 15, 491-502 (2016).

57. Eibl, G. \& Rozengurt, E. KRAS, YAP, and obesity in pancreatic cancer: a signaling network with multiple loops. Semin. Cancer Biol. 54, 50-62 (2019).

58. Hong, D. S. et al. KRAS(G12C) inhibition with sotorasib in advanced solid tumors. N. Engl. J. Med. 383, 1207-1217 (2020).

59. Igbinigie, E., Guo, F., Jiang, S. W., Kelley, C. \& Li, J. Dkk1 involvement and its potential as a biomarker in pancreatic ductal adenocarcinoma. Clin. Chim. Acta 488, 226-234 (2019).

60. Ebrahimi, S. et al. Targeting the Akt/PI3K signaling pathway as a potential therapeutic strategy for the treatment of pancreatic cancer. Curr. Med. Chem. 24, 1321-1331 (2017).

61. Song, M., Bode, A. M., Dong, Z. \& Lee, M. H. AKT as a therapeutic target for cancer. Cancer Res. 79, 1019-1031 (2019).

62. Eser, S. et al. Selective requirement of PI3K/PDK1 signaling for Kras oncogenedriven pancreatic cell plasticity and cancer. Cancer Cell 23, 406-420 (2013).

63. Baer, R. et al. Pancreatic cell plasticity and cancer initiation induced by oncogenic Kras is completely dependent on wild-type PI 3-kinase p110a. Genes Dev. 28, 2621-2635 (2014).

64. Albury, T. M. et al. Constitutively active Akt1 cooperates with KRas(G12D) to accelerate in vivo pancreatic tumor onset and progression. Neoplasia 17, 175-182 (2015).

65. Ma, J. et al. IGF-1 mediates PTEN suppression and enhances cell invasion and proliferation via activation of the IGF-1/PI3K/Akt signaling pathway in pancreatic cancer cells. J. Surg. Res. 160, 90-101 (2010).

66. Zheng, Y. et al. Insulin-like growth factor 1 -induced enolase 2 deacetylation by HDAC3 promotes metastasis of pancreatic cancer. Signal Transduct. Target Ther. 5, 53 (2020).

67. Xu, X., Yu, Y., Zong, K., Lv, P. \& Gu, Y. Up-regulation of IGF2BP2 by multiple mechanisms in pancreatic cancer promotes cancer proliferation by activating the PI3K/Akt signaling pathway. J. Exp. Clin. Cancer Res. 38, 497 (2019). 
68. Fu, Y. et al. TMEM158 promotes pancreatic cancer aggressiveness by activation of TGF $\beta 1$ and PI3K/AKT signaling pathway. J. Cell Physiol. 235, 2761-2775 (2020).

69. Jiang, N. et al. Role of PI3K/AKT pathway in cancer: the framework of malignant behavior. Mol. Biol. Rep. 47, 4587-4629 (2020).

70. Ren, X. et al. IncRNA-PLACT1 sustains activation of NF-KB pathway through a positive feedback loop with IKBa/E2F1 axis in pancreatic cancer. Mol. Cancer 19, 35 (2020).

71. Khurana, N., Dodhiawala, P. B., Bulle, A. \& Lim, K. H. Deciphering the role of innate immune NF-KB pathway in pancreatic cancer. Cancers 12, 2675 (2020).

72. Prabhu, L., Mundade, R., Korc, M., Loehrer, P. J. \& Lu, T. Critical role of NF-KB in pancreatic cancer. Oncotarget 5, 10969-10975 (2014).

73. Taniguchi, K. \& Karin, M. NF-KB, inflammation, immunity and cancer: coming of age. Nat. Rev. Immunol. 18, 309-324 (2018).

74. Karin, M. Nuclear factor-kappaB in cancer development and progression. Nature 441, 431-436 (2006)

75. Sun, Q. et al. Pin 1 promotes pancreatic cancer progression and metastasis by activation of NF-KB-IL-18 feedback loop. Cell Prolif. 53, e12816 (2020).

76. Zhou, L. et al. MicroRNA-628-5p inhibits invasion and migration of human pancreatic ductal adenocarcinoma via suppression of the AKT/NF-kappa B pathway. J. Cell Physiol. 235, 8141-8154 (2020).

77. Garg, B. et al. NFKB in pancreatic stellate cells reduces infiltration of tumors by cytotoxic T cells and killing of cancer cells, via up-regulation of CXCL12. Gastroenterology 155, 880-891.e888 (2018).

78. Ratnam, N. M. et al. NF-KB regulates GDF-15 to suppress macrophage surveillance during early tumor development. J. Clin. Invest. 127, 3796-3809 (2017).

79. Pramanik, K. C., Makena, M. R., Bhowmick, K. \& Pandey, M. K. Advancement of NF-KB signaling pathway: a novel target in pancreatic cancer. Int. J. Mol. Sci. 19, 3890 (2018).

80. Song, Y., Tang, M. Y., Chen, W., Wang, Z. \& Wang, S. L. High JAK2 protein expression predicts a poor prognosis in patients with resectable pancreatic ductal adenocarcinoma. Dis. Markers 2020, 7656031 (2020).

81. Parker, B. S., Rautela, J. \& Hertzog, P. J. Antitumour actions of interferons: implications for cancer therapy. Nat. Rev. Cancer 16, 131-144 (2016).

82. Doi, T. et al. The JAK/STAT pathway is involved in the upregulation of PD-L1 expression in pancreatic cancer cell lines. Oncol. Rep. 37, 1545-1554 (2017).

83. Lu, C., Talukder, A., Savage, N. M., Singh, N. \& Liu, K. JAK-STAT-mediated chronic inflammation impairs cytotoxic T lymphocyte activation to decrease anti-PD-1 immunotherapy efficacy in pancreatic cancer. Oncoimmunology 6, e1291106 (2017).

84. Biffi, G. et al. IL1-induced JAK/STAT signaling is antagonized by TGF $\beta$ to shape CAF heterogeneity in pancreatic ductal adenocarcinoma. Cancer Discov. 9, 282-301 (2019).

85. Mao, W., Mai, J., Peng, H., Wan, J. \& Sun, T. YAP in pancreatic cancer: oncogenic role and therapeutic strategy. Theranostics 11, 1753-1762 (2021).

86. Salcedo Allende, M. T. et al. Overexpression of Yes associated protein 1, an independent prognostic marker in patients with pancreatic ductal adenocarcinoma, correlated with liver metastasis and poor prognosis. Pancreas 46, 913-920 (2017).

87. Zhang, W. et al. Downstream of mutant KRAS, the transcription regulator YAP is essential for neoplastic progression to pancreatic ductal adenocarcinoma. Sci. Signal. 7, ra42 (2014).

88. Gruber, R. et al. YAP1 and TAZ control pancreatic cancer initiation in mice by direct up-regulation of JAK-STAT3 signaling. Gastroenterology 151, 526-539 (2016).

89. Zhang, Q. et al. Fbxw7 deletion accelerates Kras(G12D)-driven pancreatic tumorigenesis via Yap accumulation. Neoplasia 18, 666-673 (2016).

90. Murakami, S. et al. A Yap-Myc-Sox2-p53 regulatory network dictates metabolic homeostasis and differentiation in Kras-driven pancreatic ductal adenocarcinomas. Dev. Cell 51, 113-128.e119 (2019).

91. Kapoor, A. et al. Yap1 activation enables bypass of oncogenic kras addiction in pancreatic cancer. Cell 179, 1239 (2019).

92. Tu, B. et al. YAP1 oncogene is a context-specific driver for pancreatic ductal adenocarcinoma. JCl Insight 4, e130811 (2019).

93. Shao, D. D. et al. KRAS and YAP1 converge to regulate EMT and tumor survival. Cell 158, 171-184 (2014)

94. Murakami, S. et al. Yes-associated protein mediates immune reprogramming in pancreatic ductal adenocarcinoma. Oncogene 36, 1232-1244 (2017).

95. Liu, J. et al. Activation of the intrinsic fibroinflammatory program in adult pancreatic acinar cells triggered by Hippo signaling disruption. PLoS Biol. 17, e3000418 (2019).

96. Jiang, Z. et al. Inhibiting YAP expression suppresses pancreatic cancer progression by disrupting tumor-stromal interactions. J. Exp. Clin. Cancer Res. 37, 69 (2018).

97. Ram Makena, M. et al. Wnt/ $\beta$-catenin signaling: the culprit in pancreatic carcinogenesis and therapeutic resistance. Int. J. Mol. Sci. 20, 4242 (2019).
98. Nakamoto, M. \& Hisaoka, M. Clinicopathological implications of wingless/int1 (WNT) signaling pathway in pancreatic ductal adenocarcinoma. J. UOEH 38, 1-8 (2016).

99. Morris, J. P. T., Cano, D. A., Sekine, S., Wang, S. C. \& Hebrok, M. Beta-catenin blocks Kras-dependent reprogramming of acini into pancreatic cancer precursor lesions in mice. J. Clin. Invest. 120, 508-520 (2010).

100. Manegold, P. et al. Differentiation therapy targeting the $\beta$-Catenin/CBP interaction in pancreatic cancer. Cancers 10, 95 (2018)

101. Ercan, G., Karlitepe, A. \& Ozpolat, B. Pancreatic cancer stem cells and therapeutic approaches. Anticancer Res. 37, 2761-2775 (2017).

102. Zhang, Y. et al. Canonical wnt signaling is required for pancreatic carcinogenesis. Cancer Res 73, 4909-4922 (2013).

103. $\mathrm{Li}, \mathrm{H}$. et al. WD repeat-containing protein 1 maintains $\beta$-catenin activity to promote pancreatic cancer aggressiveness. Br. J. Cancer 123, 1012-1023 (2020).

104. Jiang, $\mathrm{H}$. et al. Activation of the Wnt pathway through Wnt2 promotes metastasis in pancreatic cancer. Am. J. Cancer Res. 4, 537-544 (2014).

105. Bo, H., Gao, L., Chen, Y., Zhang, J. \& Zhu, M. Upregulation of the expression of Wnt5a promotes the proliferation of pancreatic cancer cells in vitro and in a nude mouse model. Mol. Med. Rep. 13, 1163-1171 (2016).

106. Liu, $X$. et al. MUC1 regulates cyclin D1 gene expression through p120 catenin and $\beta$-catenin. Oncogenesis 3, e107 (2014).

107. Zhi, X. et al. MUC4-induced nuclear translocation of $\beta$-catenin: a novel mechanism for growth, metastasis and angiogenesis in pancreatic cancer. Cancer Lett. 346, 104-113 (2014).

108. Chartier, C. et al. Therapeutic targeting of tumor-derived R-spondin attenuates $\beta$-catenin signaling and tumorigenesis in multiple cancer types. Cancer Res 76, 713-723 (2016).

109. Criscimanna, A. et al. PanIN-specific regulation of Wnt signaling by HIF2a during early pancreatic tumorigenesis. Cancer Res. 73, 4781-4790 (2013).

110. Zhang, Q. et al. Hypoxia-inducible factor-2a promotes tumor progression and has crosstalk with Wnt/ß-catenin signaling in pancreatic cancer. Mol. Cancer 16, 119 (2017).

111. Beans, C. News feature: targeting metastasis to halt cancer's spread. Proc. Natl Acad. Sci. USA 115, 12539-12543 (2018).

112. Hegde, $\mathrm{S}$. et al. Dendritic cell paucity leads to dysfunctional immune surveillance in pancreatic cancer. Cancer Cell 37, 289-307.e289 (2020).

113. Grünwald, B. et al. Pancreatic premalignant lesions secrete tissue inhibitor of metalloproteinases-1, which activates hepatic stellate cells via CD63 signaling to create a premetastatic niche in the liver. Gastroenterology 151, 1011-1024. e1017 (2016).

114. Stratton, M. R. Exploring the genomes of cancer cells: progress and promise. Science 331, 1553-1558 (2011).

115. Campbell, P. J. et al. The patterns and dynamics of genomic instability in metastatic pancreatic cancer. Nature 467, 1109-1113 (2010).

116. Yachida, S. et al. Distant metastasis occurs late during the genetic evolution of pancreatic cancer. Nature 467, 1114-1117 (2010).

117. Guo, X. et al. Circular RNA circBFAR promotes the progression of pancreatic ductal adenocarcinoma via the miR-34b-5p/MET/Akt axis. Mol. Cancer 19, 83 (2020).

118. Cui, L. et al. Small nucleolar noncoding RNA SNORA23, up-regulated in human pancreatic ductal adenocarcinoma, regulates expression of spectrin repeatcontaining nuclear envelope 2 to promote growth and metastasis of xenograft tumors in mice. Gastroenterology 153, 292-306.e292 (2017).

119. Guo, K. et al. The novel KLF4/MSI2 signaling pathway regulates growth and metastasis of pancreatic cancer. Clin. Cancer Res. 23, 687-696 (2017).

120. He, P., Yang, J. W., Yang, V. W. \& Bialkowska, A. B. Krüppel-like factor 5, Increased in pancreatic ductal adenocarcinoma, promotes proliferation, acinar-to-ductal metaplasia, pancreatic intraepithelial neoplasia, and tumor growth in mice. Gastroenterology 154, 1494-1508.e1413 (2018).

121. Gupta, R. et al. KLF7 promotes pancreatic cancer growth and metastasis by upregulating ISG expression and maintaining Golgi complex integrity. Proc. Natl Acad. Sci. USA 117, 12341-12351 (2020).

122. Kong, Y. et al. circNFIB1 inhibits lymphangiogenesis and lymphatic metastasis via the miR-486-5p/PIK3R1/VEGF-C axis in pancreatic cancer. Mol. Cancer 19, 82 (2020).

123. Tiwari, A. et al. Loss of HIF1A from pancreatic cancer cells increases expression of PPP1R1B and degradation of $\mathrm{p} 53$ to promote invasion and metastasis. Gastroenterology 159, 1882-1897.e1885 (2020).

124. Roe, J. S. et al. Enhancer reprogramming promotes pancreatic cancer metastasis. Cell 170, 875-888.e820 (2017).

125. Argentiero, A. et al. Bone metastasis as primary presentation of pancreatic ductal adenocarcinoma: a case report and literature review. Clin. Case Rep. 7, 1972-1976 (2019).

126. Wen, J. et al. The Eighth Edition of the American Joint Committee on Cancer Distant Metastases Stage Classification for Metastatic Pancreatic 
Neuroendocrine Tumors Might Be Feasible for Metastatic Pancreatic Ductal Adenocarcinomas. Neuroendocrinology 110, 364-376 (2020).

127. Avula, L. R., Hagerty, B. \& Alewine, C. Molecular mediators of peritoneal metastasis in pancreatic cancer. Cancer Metastasis Rev. 39, 1223-1243 (2020).

128. Lee, J. W. et al. Hepatocytes direct the formation of a pro-metastatic niche in the liver. Nature 567, 249-252 (2019).

129. Maddipati, R. \& Stanger, B. Z. Pancreatic cancer metastases harbor evidence of polyclonality. Cancer Discov. 5, 1086-1097 (2015).

130. Tsai, S. et al. A serum-induced transcriptome and serum cytokine signature obtained at diagnosis correlates with the development of early pancreatic ductal adenocarcinoma metastasis. Cancer Epidemiol. Biomark. Prev. 28, 680-689 (2019).

131. Pausch, T. M. et al. Metastasis-associated fibroblasts promote angiogenesis in metastasized pancreatic cancer via the CXCL8 and the CCL2 axes. Sci. Rep. 10, 5420 (2020)

132. Lowder, C. Y. et al. Clinical implications of extensive lymph node metastases for resected pancreatic cancer. Ann. Surg. Oncol. 25, 4004-4011 (2018).

133. Nishiwada, S. et al. A microRNA signature identifies pancreatic ductal adenocarcinoma patients at risk for lymph node metastases. Gastroenterology 159 562-574 (2020).

134. Sun, W., Ren, Y., Lu, Z. \& Zhao, X. The potential roles of exosomes in pancreatic cancer initiation and metastasis. Mol. Cancer 19, 135 (2020).

135. Costa-Silva, B. et al. Pancreatic cancer exosomes initiate pre-metastatic niche formation in the liver. Nat. Cell Biol. 17, 816-826 (2015).

136. Hoshino, A. et al. Tumour exosome integrins determine organotropic metastasis. Nature 527, 329-335 (2015).

137. Zhou, P. et al. NMIIA promotes tumor growth and metastasis by activating the Wnt/ß-catenin signaling pathway and EMT in pancreatic cancer. Oncogene $\mathbf{3 8}$, 5500-5515 (2019)

138. Krebs, A. M. et al. The EMT-activator Zeb1 is a key factor for cell plasticity and promotes metastasis in pancreatic cancer. Nat. Cell Biol. 19, 518-529 (2017).

139. Yamada, S. et al. Epithelial-to-mesenchymal transition predicts prognosis of pancreatic cancer. Surgery 154, 946-954 (2013).

140. Ellenrieder, V. et al. Transforming growth factor beta 1 treatment leads to an epithelial-mesenchymal transdifferentiation of pancreatic cancer cells requiring extracellular signal-regulated kinase 2 activation. Cancer Res. 61, 4222-4228 (2001).

141. Sato, M. et al. BACH1 promotes pancreatic cancer metastasis by repressing epithelial genes and enhancing epithelial-mesenchymal transition. Cancer Res. 80, 1279-1292 (2020).

142. Niu, N. et al. Loss of Setd2 promotes Kras-induced acinar-to-ductal metaplasia and epithelia-mesenchymal transition during pancreatic carcinogenesis. Gut 69, 715-726 (2020).

143. Zheng, X. et al. Epithelial-to-mesenchymal transition is dispensable for metastasis but induces chemoresistance in pancreatic cancer. Nature 527, 525-530 (2015).

144. Satoh, K., Hamada, S. \& Shimosegawa, T. Involvement of epithelial to mesenchymal transition in the development of pancreatic ductal adenocarcinoma. J. Gastroenterol. 50, 140-146 (2015).

145. Fink, D. M., Steele, M. M. \& Hollingsworth, M. A. The lymphatic system and pancreatic cancer. Cancer Lett. 381, 217-236 (2016).

146. Ray, K. Pancreatic cancer: pancreatic cancer exosomes prime the liver for metastasis. Nat. Rev. Gastroenterol. Hepatol. 12, 371 (2015).

147. Giovannetti, E. et al. Never let it go: stopping key mechanisms underlying metastasis to fight pancreatic cancer. Semin. Cancer Biol. 44, 43-59 (2017).

148. Griesmann, H. et al. Pharmacological macrophage inhibition decreases metastasis formation in a genetic model of pancreatic cancer. Gut 66, 1278-1285 (2017).

149. Huang, C. et al. Interleukin 35 expression correlates with microvessel density in pancreatic ductal adenocarcinoma, recruits monocytes, and promotes growth and angiogenesis of xenograft tumors in mice. Gastroenterology 154, 675-688 (2018).

150. Hanahan, D. \& Weinberg, R. A. Hallmarks of cancer: the next generation. Cell 144, 646-674 (2011).

151. Sousa, C. M. \& Kimmelman, A. C. The complex landscape of pancreatic cancer metabolism. Carcinogenesis 35, 1441-1450 (2014).

152. Yang, J. et al. The enhancement of glycolysis regulates pancreatic cancer metastasis. Cell Mol. Life Sci. 77, 305-321 (2020).

153. Halbrook, C. J. \& Lyssiotis, C. A. Employing metabolism to improve the diagnosis and treatment of pancreatic cancer. Cancer Cell 31, 5-19 (2017).

154. Karasinska, J. M. et al. Altered gene expression along the glycolysis-cholesterol synthesis axis is associated with outcome in pancreatic cancer. Clin. Cancer Res 26, 135-146 (2020).

155. Hsu, P. P. \& Sabatini, D. M. Cancer cell metabolism: Warburg and beyond. Cell 134, 703-707 (2008)
156. Ying, $\mathrm{H}$. et al. Oncogenic Kras maintains pancreatic tumors through regulation of anabolic glucose metabolism. Cell 149, 656-670 (2012).

157. Bryant, K. L., Mancias, J. D., Kimmelman, A. C. \& Der, C. J. KRAS: feeding pan creatic cancer proliferation. Trends Biochem. Sci. 39, 91-100 (2014).

158. Li, X. et al. Mitochondria-translocated PGK1 functions as a protein kinase to coordinate glycolysis and the TCA cycle in tumorigenesis. Mol. Cell 61, 705-719 (2016).

159. Taparra, K. et al. O-GlcNAcylation is required for mutant KRAS-induced lung tumorigenesis. J. Clin. Invest. 128, 4924-4937 (2018).

160. Santana-Codina, N. et al. Oncogenic KRAS supports pancreatic cancer through regulation of nucleotide synthesis. Nat. Commun. 9, 4945 (2018).

161. Vernucci, E. et al. Metabolic alterations in pancreatic cancer progression. Cancers 12, 2 (2019).

162. Nagarajan, A. et al. Paraoxonase 2 facilitates pancreatic cancer growth and metastasis by stimulating GLUT1-mediated glucose transport. Mol. cell 67, 685-701 e686 (2017).

163. Zhang, C. et al. Tumour-associated mutant p53 drives the Warburg effect. Nat Commun. 4, 2935 (2013).

164. Wang, Y. P. et al. Arginine methylation of MDH1 by CARM1 inhibits glutamine metabolism and suppresses pancreatic cancer. Mol. Cell 64, 673-687 (2016).

165. McDonald, P. C. et al. Regulation of pH by carbonic anhydrase 9 mediates survival of pancreatic cancer cells with activated KRAS in response to hypoxia. Gastroenterology 157, 823-837 (2019).

166. Chaika, N. V. et al. MUC1 mucin stabilizes and activates hypoxia-inducible factor 1 alpha to regulate metabolism in pancreatic cancer. Proc. Natl Acad. Sci. USA 109, 13787-13792 (2012).

167. Shi, M. et al. A novel KLF4/LDHA signaling pathway regulates aerobic glycolysis in and progression of pancreatic cancer. Clin. Cancer Res. 20, 4370-4380 (2014).

168. Guillaumond, F. et al. Strengthened glycolysis under hypoxia supports tumor symbiosis and hexosamine biosynthesis in pancreatic adenocarcinoma. Proc Natl Acad. Sci. USA 110, 3919-3924 (2013).

169. Xu, R. et al. Reprogramming of amino acid metabolism in pancreatic cancer: recent advances and therapeutic strategies. Front. Oncol. 10, 572722 (2020).

170. Kaira, K. et al. Prognostic significance of L-type amino-acid transporter 1 expression in surgically resected pancreatic cancer. Br. J. Cancer 107, 632-638 (2012).

171. Hensley, C. T., Wasti, A. T. \& DeBerardinis, R. J. Glutamine and cancer: cell biology, physiology, and clinical opportunities. J. Clin. Investig. 123, 3678-3684 (2013).

172. Bernfeld, E. \& Foster, D. A. Glutamine as an essential amino acid for KRas-driven cancer cells. Trends Endocrinol. Metab. 30, 357-368 (2019)

173. Son, J. et al. Glutamine supports pancreatic cancer growth through a KRASregulated metabolic pathway. Nature 496, 101-105 (2013).

174. Biancur, D. E. et al. Compensatory metabolic networks in pancreatic cancers upon perturbation of glutamine metabolism. Nat. Commun. 8, 15965 (2017).

175. Udupa, S. et al. Upregulation of the glutaminase II pathway contributes to glutamate production upon glutaminase 1 inhibition in pancreatic cancer. Proteomics 19, e1800451 (2019).

176. Lampson, B. L. et al. Targeting eNOS in pancreatic cancer. Cancer Res. 72, 4472-4482 (2012)

177. Wang, H., Li, Q. F., Chow, H. Y., Choi, S. C. \& Leung, Y. C. Arginine deprivation inhibits pancreatic cancer cell migration, invasion and EMT via the down regulation of Snail, Slug, Twist, and MMP1/9. J. Physiol. Biochem. 76, 73-83 (2020).

178. Goberdhan, D. C., Wilson, C. \& Harris, A. L. Amino acid sensing by mTORC1: intracellular transporters mark the spot. Cell Metab. 23, 580-589 (2016).

179. Wang, S. et al. Metabolism. Lysosomal amino acid transporter SLC38A9 signals arginine sufficiency to mTORC1. Science 347, 188-194 (2015).

180. Wyant, G. A. et al. mTORC1 activator SLC38A9 is required to efflux essential amino acids from lysosomes and use protein as a nutrient. Cell 171, 642-654. e612 (2017).

181. Burke, L. et al. The Janus-like role of proline metabolism in cancer. Cell Death Discov. 6, 104 (2020).

182. Olivares, O. et al. Collagen-derived proline promotes pancreatic ductal adenocarcinoma cell survival under nutrient limited conditions. Nat. Commun. 8, 16031 (2017).

183. Kottakis, F. et al. LKB1 loss links serine metabolism to DNA methylation and tumorigenesis. Nature 539, 390-395 (2016).

184. Mayers, J. R. et al. Tissue of origin dictates branched-chain amino acid metabolism in mutant Kras-driven cancers. Science 353, 1161-1165 (2016).

185. Yue, M., Jiang, J., Gao, P., Liu, H. \& Qing, G. Oncogenic MYC activates a feedforward regulatory loop promoting essential amino acid metabolism and tumorigenesis. Cell Rep. 21, 3819-3832 (2017).

186. Dong, Y., Tu, R., Liu, H. \& Qing, G. Regulation of cancer cell metabolism: oncogenic MYC in the driver's seat. Signal Transduct. Target Ther. 5, 124 (2020). 
187. Mayers, J. R. et al. Elevation of circulating branched-chain amino acids is an early event in human pancreatic adenocarcinoma development. Nat. Med. 20, 1193-1198 (2014).

188. Dey, P. et al. Genomic deletion of malic enzyme 2 confers collateral lethality in pancreatic cancer. Nature 542, 119-123 (2017).

189. Röhrig, F. \& Schulze, A. The multifaceted roles of fatty acid synthesis in cancer. Nat. Rev. Cancer 16, 732-749 (2016).

190. Silvente-Poirot, S. \& Poirot, M. Cancer. Cholesterol and cancer, in the balance. Science 343, 1445-1446 (2014).

191. Philip, B. et al. A high-fat diet activates oncogenic Kras and COX2 to induce development of pancreatic ductal adenocarcinoma in mice. Gastroenterology 145, 1449-1458 (2013).

192. Guillaumond, F. et al. Cholesterol uptake disruption, in association with chemotherapy, is a promising combined metabolic therapy for pancreatic adenocarcinoma. Proc. Natl Acad. Sci. USA 112, 2473-2478 (2015).

193. Tadros, S. et al. De novo lipid synthesis facilitates gemcitabine resistance through endoplasmic reticulum stress in pancreatic cancer. Cancer Res. 77, 5503-5517 (2017).

194. Yu, M. et al. Four types of fatty acids exert differential impact on pancreatic cancer growth. Cancer Lett. 360, 187-194 (2015).

195. Ding, Y. et al. Omega-3 fatty acids prevent early pancreatic carcinogenesis via repression of the AKT pathway. Nutrients 10, 1289 (2018).

196. Snaebjornsson, M. T., Janaki-Raman, S. \& Schulze, A. Greasing the wheels of the cancer machine: the role of lipid metabolism in cancer. Cell Metab. 31, 62-76 (2020).

197. Ma, Y. et al. Fatty acid oxidation: An emerging facet of metabolic transformation in cancer. Cancer Lett. 435, 92-100 (2018).

198. De Oliveira, M. P. \& Liesa, M. The role of mitochondrial fat oxidation in cancer cell proliferation and survival. Cells 9, 2600 (2020).

199. Lee, J. S. et al. ATP production relies on fatty acid oxidation rather than glycolysis in pancreatic ductal adenocarcinoma. Cancers 12, 2477 (2020).

200. Rozeveld, C. N., Johnson, K. M., Zhang, L. \& Razidlo, G. L. KRAS controls pancreatic cancer cell lipid metabolism and invasive potential through the lipase HSL. Cancer Res. 80, 4932-4945 (2020).

201. Man, J., Pajic, M. \& Joshua, A. M. Fats and Mets, KRAS-driven lipid dysregulation affects metastatic potential in pancreatic cancer. Cancer Res. 80, 4886-4887 (2020).

202. Gabitova-Cornell, L. et al. Cholesterol pathway inhibition induces TGF- $\beta$ signaling to promote basal differentiation in pancreatic cancer. Cancer Cell $\mathbf{3 8}$, 567-583.e511 (2020)

203. Freed-Pastor, W. A. et al. Mutant p53 disrupts mammary tissue architecture via the mevalonate pathway. Cell 148, 244-258 (2012).

204. Moon, S. H. et al. p53 represses the mevalonate pathway to mediate tumor suppression. Cell 176, 564-580.e519 (2019).

205. McGregor, G. H. et al. Targeting the metabolic response to statin-mediated oxidative stress produces a synergistic antitumor response. Cancer Res. 80, 175-188 (2020).

206. Oni, T. E. et al. SOAT1 promotes mevalonate pathway dependency in pancreatic cancer. J. Exp. Med. 217, e20192389 (2020).

207. New, M. et al. Molecular pathways controlling autophagy in pancreatic cancer. Front. Oncol. 7, 28 (2017).

208. Yang, A. et al. Autophagy sustains pancreatic cancer growth through both cellautonomous and nonautonomous mechanisms. Cancer Discov. 8, 276-287 (2018).

209. Bryant, K. L. et al. Combination of ERK and autophagy inhibition as a treatment approach for pancreatic cancer. Nat. Med 25, 628-640 (2019).

210. Kinsey, C. G. et al. Protective autophagy elicited by RAF $\rightarrow$ MEK $\rightarrow$ ERK inhibition suggests a treatment strategy for RAS-driven cancers. Nat. Med. 25, 620-627 (2019).

211. Kimmelman, A. C. \& White, E. Autophagy and tumor metabolism. Cell Metab. 25, 1037-1043 (2017).

212. Perera, R. M. et al. Transcriptional control of autophagy-lysosome function drives pancreatic cancer metabolism. Nature 524, 361-365 (2015).

213. Yang, S. et al. Pancreatic cancers require autophagy for tumor growth. Genes Dev. 25, 717-729 (2011).

214. Santana-Codina, N., Mancias, J. D. \& Kimmelman, A. C. The role of autophagy in cancer. Annu. Rev. Cancer Biol. 1, 19-39 (2017).

215. Yamamoto, K. et al. Autophagy promotes immune evasion of pancreatic cancer by degrading MHC-I. Nature 581, 100-105 (2020).

216. Yamamoto, K., Venida, A., Perera, R. M. \& Kimmelman, A. C. Selective autophagy of MHC-I promotes immune evasion of pancreatic cancer. Autophagy 16, 1524-1525 (2020).

217. Ansari, D., Carvajo, M., Bauden, M. \& Andersson, R. Pancreatic cancer stroma: controversies and current insights. Scand. J. Gastroenterol. 52, 641-646 (2017).
218. Rhim, A. D. et al. Stromal elements act to restrain, rather than support, pancreatic ductal adenocarcinoma. Cancer Cell 25, 735-747 (2014).

219. Ren, B. et al. Tumor microenvironment participates in metastasis of pancreatic cancer. Mol. Cancer 17, 108 (2018).

220. Sherman, M. H. Stellate cells in tissue repair, inflammation, and cancer. Annu. Rev. Cell Dev. Biol. 34, 333-355 (2018).

221. Fu, Y., Liu, S., Zeng, S. \& Shen, H. The critical roles of activated stellate cellsmediated paracrine signaling, metabolism and onco-immunology in pancreatic ductal adenocarcinoma. Mol. Cancer 17, 62 (2018).

222. Farajzadeh Valilou, S., Keshavarz-Fathi, M., Silvestris, N., Argentiero, A. \& Rezaei, $\mathrm{N}$. The role of inflammatory cytokines and tumor associated macrophages (TAMs) in microenvironment of pancreatic cancer. Cytokine Growth Factor Rev. 39, 46-61 (2018).

223. Yoshikawa, K. et al. Impact of tumor-associated macrophages on invasive ductal carcinoma of the pancreas head. Cancer Sci. 103, 2012-2020 (2012).

224. Jang, J. E. et al. Crosstalk between regulatory $T$ cells and tumor-associated dendritic cells negates anti-tumor immunity in pancreatic cancer. Cell Rep. 20, 558-571 (2017).

225. Zhang, Y. et al. Regulatory T-cell depletion alters the tumor microenvironment and accelerates pancreatic carcinogenesis. Cancer Discov. 10, 422-439 (2020).

226. Thyagarajan, A. et al. Myeloid-derived suppressor cells and pancreatic cancer: implications in novel therapeutic approaches. Cancers 11, 1627 (2019).

227. Pergamo, M. \& Miller, G. Myeloid-derived suppressor cells and their role in pancreatic cancer. Cancer Gene Ther. 24, 100-105 (2017).

228. von Ahrens, D., Bhagat, T. D., Nagrath, D., Maitra, A. \& Verma, A. The role of stromal cancer-associated fibroblasts in pancreatic cancer. J. Hematol. Oncol. 10, 76 (2017).

229. Pothula, S. P. et al. Key role of pancreatic stellate cells in pancreatic cancer. Cancer Lett. 381, 194-200 (2016).

230. Bulle, A. \& Lim, K. H. Beyond just a tight fortress: contribution of stroma to epithelial-mesenchymal transition in pancreatic cancer. Signal Transduct. Target Ther. 5, 249 (2020).

231. Leca, J. et al. Cancer-associated fibroblast-derived annexin A6+ extracellular vesicles support pancreatic cancer aggressiveness. J. Clin. Invest. 126, 4140-4156 (2016).

232. Zhao, $\mathrm{H}$. et al. Tumor microenvironment derived exosomes pleiotropically modulate cancer cell metabolism. Elife 5, e10250 (2016).

233. Richards, K. E. et al. Cancer-associated fibroblast exosomes regulate survival and proliferation of pancreatic cancer cells. Oncogene 36, 1770-1778 (2017).

234. Lo, A. et al. Fibroblast activation protein augments progression and metastasis of pancreatic ductal adenocarcinoma. JCl Insight 2, e92232 (2017).

235. Chen, S. et al. MiR-21-mediated metabolic alteration of cancer-associated fibroblasts and its effect on pancreatic cancer cell behavior. Int. J. Biol. Sci. 14, 100-110 (2018).

236. Sousa, C. M. et al. Pancreatic stellate cells support tumour metabolism through autophagic alanine secretion. Nature 536, 479-483 (2016)

237. Auciello, F. R. et al. A stromal lysolipid-autotaxin signaling axis promotes pancreatic tumor progression. Cancer Discov. 9, 617-627 (2019).

238. Shindo, K. et al. Podoplanin expression in cancer-associated fibroblasts enhances tumor progression of invasive ductal carcinoma of the pancreas. Mol. Cancer 12, 168 (2013).

239. Feig, C. et al. Targeting CXCL12 from FAP-expressing carcinoma-associated fibroblasts synergizes with anti-PD-L1 immunotherapy in pancreatic cancer. Proc. Natl Acad. Sci. USA 110, 20212-20217 (2013).

240. Hwang, R. F. et al. Inhibition of the hedgehog pathway targets the tumorassociated stroma in pancreatic cancer. Mol. Cancer Res. 10, 1147-1157 (2012).

241. Laklai, H. et al. Genotype tunes pancreatic ductal adenocarcinoma tissue tension to induce matricellular fibrosis and tumor progression. Nat. Med. 22, 497-505 (2016).

242. Tape, C. J. et al. Oncogenic KRAS regulates tumor cell signaling via stromal reciprocation. Cell 165, 910-920 (2016).

243. Ho, W. J., Jaffee, E. M. \& Zheng, L. The tumour microenvironment in pancreatic cancer - clinical challenges and opportunities. Nat. Rev. Clin. Oncol. 17, 527-540 (2020).

244. Vonderheide, R. H. \& Bayne, L. J. Inflammatory networks and immune surveillance of pancreatic carcinoma. Curr. Opin. Immunol. 25, 200-205 (2013).

245. Meyer, M. A. et al. Breast and pancreatic cancer interrupt IRF8-dependent dendritic cell development to overcome immune surveillance. Nat. Commun. 9, 1250 (2018).

246. Tjomsland, V. et al. The desmoplastic stroma plays an essential role in the accumulation and modulation of infiltrated immune cells in pancreatic adenocarcinoma. Clin. Dev. Immunol. 2011, 212810 (2011).

247. Mahajan, U. M. et al. Immune cell and stromal signature associated with progression-free survival of patients with resected pancreatic ductal adenocarcinoma. Gastroenterology 155, 1625-1639.e1622 (2018). 
248. Carstens, J. L. et al. Spatial computation of intratumoral T cells correlates with survival of patients with pancreatic cancer. Nat. Commun. 8, 15095 (2017).

249. Pylayeva-Gupta, Y., Lee, K. E., Hajdu, C. H., Miller, G. \& Bar-Sagi, D. Oncogenic Kras-induced GM-CSF production promotes the development of pancreatic neoplasia. Cancer Cell 21, 836-847 (2012).

250. Sivaram, N. et al. Tumor-intrinsic PIK3CA represses tumor immunogenecity in a model of pancreatic cancer. J. Clin. Invest. 129, 3264-3276 (2019).

251. Hashimoto, S. et al. ARF6 and AMAP1 are major targets of KRAS and TP53 mutations to promote invasion, PD-L1 dynamics, and immune evasion of pancreatic cancer. Proc. Natl Acad. Sci. USA 116, 17450-17459 (2019).

252. Wörmann, S. M. et al. Loss of P53 function activates JAK2-STAT3 signaling to promote pancreatic tumor growth, stroma modification, and gemcitabine resistance in mice and is associated with patient survival. Gastroenterology 151, 180-193.e112 (2016).

253. Serrels, A. et al. Nuclear FAK controls chemokine transcription, Tregs, and evasion of anti-tumor immunity. Cell 163, 160-173 (2015).

254. Jiang, $H$. et al. Targeting focal adhesion kinase renders pancreatic cancers responsive to checkpoint immunotherapy. Nat. Med. 22, 851-860 (2016).

255. Sodir, N. M. et al. Endogenous Myc maintains the tumor microenvironment. Genes Dev. 25, 907-916 (2011).

256. Sodir, N. M. et al. MYC instructs and maintains pancreatic adenocarcinoma phenotype. Cancer Discov. 10, 588-607 (2020).

257. Muthalagu, N. et al. Repression of the type I interferon pathway underlies MYC and KRAS-dependent evasion of NK and B cells in pancreatic ductal adenocarcinoma. Cancer Discov. 10, 872-887 (2020).

258. Dovmark, T. H., Saccomano, M., Hulikova, A., Alves, F. \& Swietach, P. Connexin-43 channels are a pathway for discharging lactate from glycolytic pancreatic ductal adenocarcinoma cells. Oncogene 36, 4538-4550 (2017).

259. Ye, $\mathrm{H}$. et al. Tumor-associated macrophages promote progression and the Warburg effect via CCL18/NF-kB/NCAM-1 pathway in pancreatic ductal adenocarcinoma. Cell Death Dis. 9, 453 (2018).

260. Hutcheson, J. et al. Immunologic and metabolic features of pancreatic ductal adenocarcinoma define prognostic subtypes of disease. Clin. Cancer Res. 22, 3606-3617 (2016)

261. Penny, H. L. et al. Warburg metabolism in tumor-conditioned macrophages promotes metastasis in human pancreatic ductal adenocarcinoma. Oncoimmunology 5, e1191731 (2016).

262. Wang, X. et al. Cancer-FOXP3 directly activated CCL5 to recruit FOXP3(+)Treg cells in pancreatic ductal adenocarcinoma. Oncogene 36, 3048-3058 (2017).

263. Wang, X. et al. PD-L1 is a direct target of cancer-FOXP3 in pancreatic ductal adenocarcinoma (PDAC), and combined immunotherapy with antibodies against PD-L1 and CCL5 is effective in the treatment of PDAC. Signal Transduct. Target Ther. 5, 38 (2020)

264. Zhu, Y. et al. CSF1/CSF1R blockade reprograms tumor-infiltrating macrophages and improves response to T-cell checkpoint immunotherapy in pancreatic cancer models. Cancer Res. 74, 5057-5069 (2014).

265. ljichi, H. et al. Inhibiting $\mathrm{Cxcr} 2$ disrupts tumor-stromal interactions and improves survival in a mouse model of pancreatic ductal adenocarcinoma. J. Clin. Invest. 121, 4106-4117 (2011).

266. Vonderheide, R. H. \& Bear, A. S. Tumor-derived myeloid cell chemoattractants and T cell exclusion in pancreatic cancer. Front Immunol. 11, 605619 (2020).

267. Sanford, D. E. et al. Inflammatory monocyte mobilization decreases patient survival in pancreatic cancer: a role for targeting the CCL2/CCR2 axis. Clin. Cancer Res. 19, 3404-3415 (2013).

268. Hou, P. et al. Tumor microenvironment remodeling enables bypass of oncogenic KRAS dependency in pancreatic cancer. Cancer Discov. 10, 1058-1077 (2020).

269. Zhang, R. et al. CXCL5 overexpression predicts a poor prognosis in pancreatic ductal adenocarcinoma and is correlated with immune cell infiltration. J. Cancer 11, 2371-2381 (2020)

270. Seifert, L. et al. The necrosome promotes pancreatic oncogenesis via CXCL1 and Mincle-induced immune suppression. Nature 532, 245-249 (2016).

271. Le, D. T. et al. Safety and survival with GVAX pancreas prime and Listeria monocytogenes-expressing mesothelin (CRS-207) boost vaccines for metastatic pancreatic cancer. J. Clin. Oncol. 33, 1325-1333 (2015).

272. Oh, M. H. et al. Targeting glutamine metabolism enhances tumor-specific immunity by modulating suppressive myeloid cells. J. Clin. Invest. 130, 3865-3884 (2020)

273. $\mathrm{Li}$, J. et al. Tumor cell-intrinsic factors underlie heterogeneity of immune cell infiltration and response to immunotherapy. Immunity 49, 178-193.e177 (2018).

274. Zhang, A. et al. Cancer-associated fibroblasts promote M2 polarization of macrophages in pancreatic ductal adenocarcinoma. Cancer Med. 6, 463-470 (2017).

275. Steins, A. et al. High-grade mesenchymal pancreatic ductal adenocarcinoma drives stromal deactivation through CSF-1. EMBO Rep. 21, e48780 (2020).
276. Principe, D. R. et al. TGF $\beta$ signaling in the pancreatic tumor microenvironment promotes fibrosis and immune evasion to facilitate tumorigenesis. Cancer Res. 76, 2525-2539 (2016).

277. Yin, Z. et al. Macrophage-derived exosomal microRNA-501-3p promotes progression of pancreatic ductal adenocarcinoma through the TGFBR3-mediated TGF- $\beta$ signaling pathway. J. Exp. Clin. Cancer Res. 38, 310 (2019).

278. Lianyuan, T. et al. Tumor associated neutrophils promote the metastasis of pancreatic ductal adenocarcinoma. Cancer Biol. Ther. 21, 937-945 (2020).

279. Melzer, M. K. et al. An immunological glance on pancreatic ductal adenocarcinoma. Int. J. Mol. Sci. 21, 3345 (2020).

280. Martinez-Bosch, N., Vinaixa, J. \& Navarro, P. Immune evasion in pancreatic cancer: from mechanisms to therapy. Cancers 10, 6 (2018).

281. Kaur, A. et al. A recombinant fragment of human surfactant protein d induces apoptosis in pancreatic cancer cell lines via Fas-mediated pathway. Front. Immunol. 9, 1126 (2018).

282. Kamisawa, T., Wood, L. D., Itoi, T. \& Takaori, K. Pancreatic cancer. Lancet $\mathbf{3 8 8}$ 73-85 (2016).

283. Jeune, F. et al. Pancreatic cancer surgical management. Presse Med. 48 e147-e158 (2019).

284. Li, H. Y., Cui, Z. M., Chen, J., Guo, X. Z. \& Li, Y. Y. Pancreatic cancer: diagnosis and treatments. Tumour Biol. 36, 1375-1384 (2015).

285. Zhang, Y. et al. Tumor markers CA19-9, CA242 and CEA in the diagnosis of pancreatic cancer: a meta-analysis. Int. J. Clin. Exp. Med. 8, 11683-11691 (2015).

286. van Manen, $L$ et al. Stage-specific value of carbohydrate antigen 19-9 and carcinoembryonic antigen serum levels on survival and recurrence in pancreatic cancer: a single center study and meta-analysis. Cancers 12, 2970 (2020).

287. Daoud, A. Z., Mulholland, E. J., Cole, G. \& McCarthy, H. O. MicroRNAs in pancreatic cancer: biomarkers, prognostic, and therapeutic modulators. BMC Cancer 19, 1130 (2019).

288. Previdi, M. C., Carotenuto, P., Zito, D., Pandolfo, R. \& Braconi, C. Noncoding RNAs as novel biomarkers in pancreatic cancer: what do we know? Fut. Oncol. 13 443-453 (2017).

289. Cicenas, J. et al. KRAS, TP53, CDKN2A, SMAD4, BRCA1, and BRCA2 mutations in pancreatic cancer. Cancers 9, 42 (2017).

290. Takai, E. \& Yachida, S. Circulating tumor DNA as a liquid biopsy target for detection of pancreatic cancer. World J. Gastroenterol. 22, 8480-8488 (2016).

291. Martini, V., Timme-Bronsert, S., Fichtner-Feigl, S., Hoeppner, J. \& Kulemann, B. Circulating tumor cells in pancreatic cancer: current perspectives. Cancers 11, 1659 (2019).

292. Nagrath, S., Jack, R. M., Sahai, V. \& Simeone, D. M. Opportunities and challenges for pancreatic circulating tumor cells. Gastroenterology 151, 412-426 (2016).

293. Heiler, S., Wang, Z. \& Zöller, M. Pancreatic cancer stem cell markers and exosomes - the incentive push. World J. Gastroenterol. 22, 5971-6007 (2016).

294. Zhao, C., Gao, F., Weng, S. \& Liu, Q. Pancreatic cancer and associated exosomes. Cancer Biomark. 20, 357-367 (2017).

295. Lee, J. S., Park, S. S., Lee, Y. K., Norton, J. A. \& Jeffrey, S. S. Liquid biopsy in pancreatic ductal adenocarcinoma: current status of circulating tumor cells and circulating tumor DNA. Mol. Oncol. 13, 1623-1650 (2019).

296. Riva, F. et al. Clinical applications of circulating tumor DNA and circulating tumor cells in pancreatic cancer. Mol. Oncol. 10, 481-493 (2016).

297. Gao, Y. et al. Clinical significance of pancreatic circulating tumor cells using combined negative enrichment and immunostaining-fluorescence in situ hybridization. J. Exp. Clin. Cancer Res. 35, 66 (2016).

298. Xu, Y. et al. Detection of circulating tumor cells using negative enrichment immunofluorescence and an in situ hybridization system in pancreatic cancer. Int. J. Mol. Sci. 18, 622 (2017).

299. Ankeny, J. S. et al. Circulating tumour cells as a biomarker for diagnosis and staging in pancreatic cancer. Br. J. Cancer 114, 1367-1375 (2016).

300. Effenberger, K. E. et al. Improved risk stratification by circulating tumor cell counts in pancreatic cancer. Clin. Cancer Res. 24, 2844-2850 (2018).

301. Poruk, K. E. et al. Circulating tumor cell phenotype predicts recurrence and survival in pancreatic adenocarcinoma. Ann. Surg. 264, 1073-1081 (2016).

302. Zhang, Y. et al. Patterns of circulating tumor cells identified by CEP8, CK and CD45 in pancreatic cancer. Int. J. Cancer 136, 1228-1233 (2015).

303. Bissolati, M. et al. Portal vein-circulating tumor cells predict liver metastases in patients with resectable pancreatic cancer. Tumour Biol. 36, 991-996 (2015).

304. Tien, Y. W. et al. A high circulating tumor cell count in portal vein predicts liver metastasis from periampullary or pancreatic cancer: a high portal venous CTC count predicts liver metastases. Medicine 95, e3407 (2016).

305. Chang, M. C. et al. Clinical significance of circulating tumor microemboli as a prognostic marker in patients with pancreatic ductal adenocarcinoma. Clin. Chem. 62, 505-513 (2016).

306. Bettegowda, C. et al. Detection of circulating tumor DNA in early- and late-stage human malignancies. Sci. Transl. Med. 6, 224 ra224 (2014). 
307. Cohen, J. D. et al. Combined circulating tumor DNA and protein biomarkerbased liquid biopsy for the earlier detection of pancreatic cancers. Proc. Natl Acad. Sci. USA 114, 10202-10207 (2017).

308. Kinugasa, H. et al. Detection of K-ras gene mutation by liquid biopsy in patients with pancreatic cancer. Cancer 121, 2271-2280 (2015).

309. Perets, R. et al. Mutant KRAS circulating tumor DNA is an accurate tool for pancreatic cancer monitoring. Oncologist 23, 566-572 (2018).

310. Pietrasz, D. et al. Plasma circulating tumor DNA in pancreatic cancer patients is a prognostic marker. Clin. Cancer Res. 23, 116-123 (2017).

311. Creemers, A. et al. Clinical value of ctDNA in upper-Gl cancers: a systematic review and meta-analysis. Biochim. Biophys. Acta Rev. Cancer 1868, 394-403 (2017).

312. Hadano, N. et al. Prognostic value of circulating tumour DNA in patients undergoing curative resection for pancreatic cancer. Br. J. Cancer 115, 59-65 (2016).

313. Singh, N., Gupta, S., Pandey, R. M., Chauhan, S. S. \& Saraya, A. High levels of cellfree circulating nucleic acids in pancreatic cancer are associated with vascular encasement, metastasis and poor survival. Cancer Invest. 33, 78-85 (2015).

314. Mashouri, L. et al. Exosomes: composition, biogenesis, and mechanisms in cancer metastasis and drug resistance. Mol. Cancer 18, 75 (2019).

315. Whiteside, T. L. Tumor-derived exosomes and their role in cancer progression. Adv. Clin. Chem. 74, 103-141 (2016).

316. Zhu, L. et al. Isolation and characterization of exosomes for cancer research. J. Hematol. Oncol. 13, 152 (2020).

317. Melo, S. A. et al. Glypican-1 identifies cancer exosomes and detects early pancreatic cancer. Nature 523, 177-182 (2015).

318. Lewis, J. M. et al. Integrated analysis of exosomal protein biomarkers on alternating current electrokinetic chips enables rapid detection of pancreatic cancer in patient blood. ACS Nano 12, 3311-3320 (2018).

319. Théry, C. Cancer: diagnosis by extracellular vesicles. Nature 523, 161-162 (2015).

320. Lai, X. et al. A microRNA signature in circulating exosomes is superior to exosomal glypican-1 levels for diagnosing pancreatic cancer. Cancer Lett. 393, 86-93 (2017).

321. Liang, K. et al. Nanoplasmonic quantification of tumor-derived extracellular vesicles in plasma microsamples for diagnosis and treatment monitoring. Nat. Biomed. Eng. 1, 0021 (2017).

322. Allenson, K. et al. High prevalence of mutant KRAS in circulating exosomederived DNA from early-stage pancreatic cancer patients. Ann. Oncol. 28, 741-747 (2017).

323. An, M. et al. Quantitative proteomic analysis of serum exosomes from patients with locally advanced pancreatic cancer undergoing chemoradiotherapy. J. Proteome Res. 16, 1763-1772 (2017).

324. Yang, K. S. et al. Multiparametric plasma EV profiling facilitates diagnosis of pancreatic malignancy. Sci. Transl. Med. 9, eaal3226 (2017).

325. Lim, M., Kim, C. J., Sunkara, V., Kim, M. H. \& Cho, Y. K. Liquid biopsy in lung cancer: clinical applications of circulating biomarkers (CTCs and ctDNA). Micromachines 9, 100 (2018).

326. Cheng, D. T. et al. Memorial Sloan Kettering-Integrated Mutation Profiling of Actionable Cancer Targets (MSK-IMPACT): a hybridization capture-based nextgeneration sequencing clinical assay for solid tumor molecular oncology. J. Mol. Diagn. 17, 251-264 (2015).

327. Razavi, P. et al. High-intensity sequencing reveals the sources of plasma circulating cell-free DNA variants. Nat. Med. 25, 1928-1937 (2019).

328. Zehir, A. et al. Mutational landscape of metastatic cancer revealed from prospective clinical sequencing of 10,000 patients. Nat. Med. 23, 703-713 (2017).

329. Samstein, R. M. et al. Tumor mutational load predicts survival after immunotherapy across multiple cancer types. Nat. Genet. 51, 202-206 (2019).

330. Law, H. C. et al. The proteomic landscape of pancreatic ductal adenocarcinoma liver metastases identifies molecular subtypes and associations with clinical response. Clin. Cancer Res. 26, 1065-1076 (2020).

331. Aung, K. L. et al. Genomics-driven precision medicine for advanced pancreatic cancer: early results from the COMPASS Trial. Clin. Cancer Res. 24, 1344-1354 (2018).

332. Hayashi, A. et al. A unifying paradigm for transcriptional heterogeneity and squamous features in pancreatic ductal adenocarcinoma. Nat. Cancer 1, 59-74 (2020).

333. Er, J. L. et al. Identification of inhibitors synergizing gemcitabine sensitivity in the squamous subtype of pancreatic ductal adenocarcinoma (PDAC). Apoptosis 23, 343-355 (2018)

334. Kalimuthu, S. N. et al. Morphological classification of pancreatic ductal adenocarcinoma that predicts molecular subtypes and correlates with clinical outcome. Gut 69, 317-328 (2020).

335. Somerville, T. D. D. et al. TP63-mediated enhancer reprogramming drives the squamous subtype of pancreatic ductal adenocarcinoma. Cell Rep. 25, 1741-1755.e1747 (2018).
336. Roa-Peña, L. et al. Keratin 17 identifies the most lethal molecular subtype of pancreatic cancer. Sci. Rep. 9, 11239 (2019).

337. Kaissis, G. A. et al. Image-based molecular phenotyping of pancreatic ductal adenocarcinoma. J. Clin. Med. 9, 724 (2020).

338. Aguirre, A. J. et al. Real-time genomic characterization of advanced pancreatic cancer to enable precision medicine. Cancer Discov. 8, 1096-1111 (2018).

339. Collisson, E. A., Bailey, P., Chang, D. K. \& Biankin, A. V. Molecular subtypes of pancreatic cancer. Nat. Rev. Gastroenterol. Hepatol. 16, 207-220 (2019).

340. Aparicio, S., Hidalgo, M. \& Kung, A. L. Examining the utility of patient-derived xenograft mouse models. Nat. Rev. Cancer 15, 311-316 (2015).

341. Huang, L. et al. Ductal pancreatic cancer modeling and drug screening using human pluripotent stem cell- and patient-derived tumor organoids. Nat. Med. 21, 1364-1371 (2015).

342. Vlachogiannis, $\mathrm{G}$. et al. Patient-derived organoids model treatment response of metastatic gastrointestinal cancers. Science 359, 920-926 (2018).

343. Hingorani, S. R. et al. Preinvasive and invasive ductal pancreatic cancer and its early detection in the mouse. Cancer Cell 4, 437-450 (2003).

344. Olive, K. P. \& Tuveson, D. A. The use of targeted mouse models for preclinical testing of novel cancer therapeutics. Clin. Cancer Res. 12, 5277-5287 (2006).

345. Hingorani, S. R. et al. Trp53R172H and KrasG12D cooperate to promote chromosomal instability and widely metastatic pancreatic ductal adenocarcinoma in mice. Cancer Cell 7, 469-483 (2005).

346. Aguirre, A. J. et al. Activated Kras and Ink4a/Arf deficiency cooperate to produce metastatic pancreatic ductal adenocarcinoma. Genes Dev. 17, 3112-3126 (2003).

347. Bardeesy, N. et al. Both p16(Ink4a) and the p19(Arf)-p53 pathway constrain progression of pancreatic adenocarcinoma in the mouse. Proc. Natl Acad. Sci. USA 103, 5947-5952 (2006).

348. Drosos, Y. et al. ATM-deficiency increases genomic instability and metastatic potential in a mouse model of pancreatic cancer. Sci. Rep. 7, 11144 (2017).

349. Russell, R. et al. Loss of ATM accelerates pancreatic cancer formation and epithelial-mesenchymal transition. Nat. Commun. 6, 7677 (2015).

350. Siveke, J. T. et al. Concomitant pancreatic activation of $\operatorname{Kras}(\mathrm{G} 12 \mathrm{D})$ and Tgfa results in cystic papillary neoplasms reminiscent of human IPMN. Cancer Cell 12, 266-279 (2007).

351. Bardeesy, N. et al. Smad4 is dispensable for normal pancreas development yet critical in progression and tumor biology of pancreas cancer. Genes Dev. 20, 3130-3146 (2006).

352. Kojima, K. et al. Inactivation of Smad4 accelerates Kras(G12D)-mediated pancreatic neoplasia. Cancer Res. 67, 8121-8130 (2007).

353. Gopinathan, A., Morton, J. P., Jodrell, D. I. \& Sansom, O. J. GEMMs as preclinical models for testing pancreatic cancer therapies. Dis. Model. Mech. 8, 1185-1200 (2015).

354. Francia, G., Cruz-Munoz, W., Man, S., Xu, P. \& Kerbel, R. S. Mouse models of advanced spontaneous metastasis for experimental therapeutics. Nat. Rev. Cancer 11, 135-141 (2011).

355. Wolff, R. A., Varadhachary, G. R. \& Evans, D. B. Adjuvant therapy for adenocarcinoma of the pancreas: analysis of reported trials and recommendations for future progress. Ann. Surg. Oncol. 15, 2773-2786 (2008).

356. Fenocchio, E. et al. Is there a standard adjuvant therapy for resected pancreatic cancer? Cancers 11, 0 (2019).

357. Merkow, R. P. et al. Postoperative complications reduce adjuvant chemotherapy use in resectable pancreatic cancer. Ann. Surg. 260, 372-377 (2014).

358. Dhir, M. et al. Neoadjuvant treatment of pancreatic adenocarcinoma: a systematic review and meta-analysis of 5520 patients. World J. Surg. Oncol. 15, 183 (2017).

359. Kim, S. et al. Comparative effectiveness of nab-paclitaxel plus gemcitabine vs FOLFIRINOX in metastatic pancreatic cancer: a retrospective nationwide chart review in the United States. Adv. Ther. 35, 1564-1577 (2018).

360. Sohal, D. P., Mangu, P. B. \& Laheru, D. Metastatic pancreatic cancer: American Society of Clinical Oncology Clinical Practice Guideline Summary. J. Oncol. Pract. 13, 261-264 (2017)

361. Sohal, D. P. S. et al. Metastatic pancreatic cancer: ASCO Clinical Practice Guideline Update. J. Clin. Oncol. 36, 2545-2556 (2018).

362. Wang-Gillam, A. et al. Nanoliposomal irinotecan with fluorouracil and folinic acid in metastatic pancreatic cancer after previous gemcitabine-based therapy (NAPOLI-1): a global, randomised, open-label, phase 3 trial. Lancet 387, 545-557 (2016).

363. Ueno, M. et al. A randomized phase II study of S-1 plus oral leucovorin versus S-1 monotherapy in patients with gemcitabine-refractory advanced pancreatic cancer. Ann. Oncol. 27, 502-508 (2016).

364. McCormick, F. KRAS as a therapeutic target. Clin. Cancer Res. 21, 1797-1801 (2015).

365. Kamerkar, S. et al. Exosomes facilitate therapeutic targeting of oncogenic KRAS in pancreatic cancer. Nature 546, 498-503 (2017). 
366. Doebele, R. C. et al. Entrectinib in patients with advanced or metastatic NTRK fusion-positive solid tumours: integrated analysis of three phase 1-2 trials. Lancet Oncol. 21, 271-282 (2020).

367. Drilon, A. et al. Efficacy of larotrectinib in TRK fusion-positive cancers in adults and children. N. Engl. J. Med. 378, 731-739 (2018).

368. Lin, J. J., Riely, G. J. \& Shaw, A. T. Targeting ALK: precision medicine takes on drug resistance. Cancer Discov. 7, 137-155 (2017).

369. Singhi, A. D. et al. Identification of targetable ALK rearrangements in pancreatic ductal adenocarcinoma. J. Natl Compr. Cancer Netw. 15, 555-562 (2017).

370. Kaufman, B. et al. Olaparib monotherapy in patients with advanced cancer and a germline BRCA1/2 mutation. J. Clin. Oncol. 33, 244-250 (2015).

371. Royal, R. E. et al. Phase 2 trial of single agent Ipilimumab (anti-CTLA-4) for locally advanced or metastatic pancreatic adenocarcinoma. J. Immunother. 33, 828-833 (2010).

372. Brahmer, J. R. et al. Safety and activity of anti-PD-L1 antibody in patients with advanced cancer. N. Engl. J. Med. 366, 2455-2465 (2012).

373. Marabelle, A. et al. Efficacy of pembrolizumab in patients with noncolorectal high microsatellite instability/mismatch repair-deficient cancer: results from the Phase II KEYNOTE-158 Study. J. Clin. Oncol. 38, 1-10 (2020).

374. Toole, B. P. \& Slomiany, M. G. Hyaluronan: a constitutive regulator of chemoresistance and malignancy in cancer cells. Semin. Cancer Biol. 18, 244-250 (2008).

375. Hingorani, S. R. et al. HALO 202: randomized phase II study of PEGPH20 plus nab-paclitaxel/gemcitabine versus nab-paclitaxel/gemcitabine in patients with untreated, metastatic pancreatic ductal adenocarcinoma. J. Clin. Oncol. 36, 359-366 (2018).

376. Van Cutsem, E. et al. Randomized phase III trial of pegvorhyaluronidase alfa with nab-paclitaxel plus gemcitabine for patients with hyaluronan-high metastatic pancreatic adenocarcinoma. J. Clin. Oncol. 38, 3185-3194 (2020).

377. Alistar, A. et al. Safety and tolerability of the first-in-class agent CPI-613 in combination with modified FOLFIRINOX in patients with metastatic pancreatic cancer: a single-centre, open-label, dose-escalation, phase 1 trial. Lancet Oncol. 18, 770-778 (2017).

378. Philip, P. A. et al. A phase III open-label trial to evaluate efficacy and safety of CPI-613 plus modified FOLFIRINOX (mFFX) versus FOLFIRINOX (FFX) in patients with metastatic adenocarcinoma of the pancreas. Fut. Oncol. 15, 3189-3196 (2019).
379. Wolpin, B. M. et al. Phase II and pharmacodynamic study of autophagy inhibition using hydroxychloroquine in patients with metastatic pancreatic adenocarcinoma. Oncologist 19, 637-638 (2014).

380. Bennewith, K. L. et al. The role of tumor cell-derived connective tissue growth factor (CTGF/CCN2) in pancreatic tumor growth. Cancer Res. 69, 775-784 (2009).

381. Neesse, A. et al. CTGF antagonism with mAb FG-3019 enhances chemotherapy response without increasing drug delivery in murine ductal pancreas cancer. Proc. Natl Acad. Sci. USA 110, 12325-12330 (2013).

382. Sonbol, M. B. et al. CanStem111P trial: a Phase III study of napabucasin plus nabpaclitaxel with gemcitabine. Fut. Oncol. 15, 1295-1302 (2019).

383. Li, C. et al. Identification of pancreatic cancer stem cells. Cancer Res. 67 1030-1037 (2007)

384. Gurlevik, E. et al. Administration of gemcitabine after pancreatic tumor resection in mice induces an antitumor immune response mediated by natural killer cells. Gastroenterology 151, 338-350.e337 (2016).

385. Izeradjene, K. et al. Kras(G12D) and Smad4/Dpc4 haploinsufficiency cooperate to induce mucinous cystic neoplasms and invasive adenocarcinoma of the pancreas. Cancer Cell 11, 229-243 (2007).

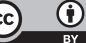

Open Access This article is licensed under a Creative Commons Attribution 4.0 International License, which permits use, sharing, adaptation, distribution and reproduction in any medium or format, as long as you give appropriate credit to the original author(s) and the source, provide a link to the Creative Commons license, and indicate if changes were made. The images or other third party material in this article are included in the article's Creative Commons license, unless indicated otherwise in a credit line to the material. If material is not included in the article's Creative Commons license and your intended use is not permitted by statutory regulation or exceeds the permitted use, you will need to obtain permission directly from the copyright holder. To view a copy of this license, visit http://creativecommons. org/licenses/by/4.0/.

(c) The Author(s) 2021 